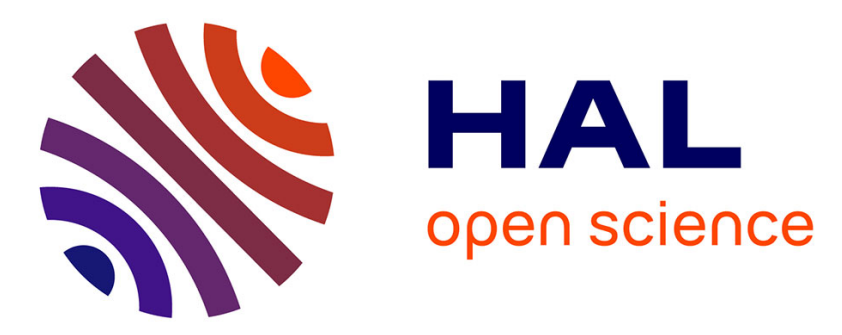

\title{
An analysis of the symmetry issue in the $\ell$-distribution method of gas radiation in non-uniform gaseous media
}

Frédéric André

\section{To cite this version:}

Frédéric André. An analysis of the symmetry issue in the $\ell$-distribution method of gas radiation in non-uniform gaseous media. Journal of Quantitative Spectroscopy and Radiative Transfer, 2017, 190, pp.78-87. 10.1016/j.jqsrt.2017.01.002 . hal-02064929

\section{HAL Id: hal-02064929 \\ https://hal.science/hal-02064929}

Submitted on 23 Apr 2019

HAL is a multi-disciplinary open access archive for the deposit and dissemination of scientific research documents, whether they are published or not. The documents may come from teaching and research institutions in France or abroad, or from public or private research centers.
L'archive ouverte pluridisciplinaire HAL, est destinée au dépôt et à la diffusion de documents scientifiques de niveau recherche, publiés ou non, émanant des établissements d'enseignement et de recherche français ou étrangers, des laboratoires publics ou privés. 


\title{
AN ANALYSIS OF THE SYMMETRY ISSUE IN THE ८- DISTRIBUTION METHOD OF GAS RADIATION IN NON-UNIFORM GASEOUS MEDIA
}

\author{
Frédéric André
}

Univ Lyon, CNRS, INSA-Lyon, Université Claude Bernard Lyon 1, CETHIL UMR5008, F-69621, Villeurbanne, France. 
Abstract. The recently proposed $\ell$-distribution / ICE (Iterative Copula Evaluation) method of gas radiation suffers from symmetry issues when applied in highly non-isothermal and nonhomogeneous gaseous media. This problem is studied in a detailed theoretical way. The objective of the present paper is: $1 /$ to provide a mathematical analysis of this problem of symmetry and, $2 /$ to suggest a decisive factor, defined in terms of the ratio between the narrow band Planck and Rosseland mean absorption coefficients, to handle this issue. Comparisons of model predictions with reference LBL calculations show that the proposed criterion improves the accuracy of the intuitive ICE method for applications in highly non-uniform gases at high temperatures.

KEYWORDS: e-distribution, Godson-Weinreb-Neuendorffer's method, Levy subordinated Archimedean copulas, symmetry. 


\section{NOMENCLATURE}

C copula

Gr rank transmutation map

$\ell \quad$ inverse of the transmission function $(\mathrm{cm})$ as defined in Section 2

L gas path length (cm)

\section{Greek symbols}

$\alpha \quad$ absorptivity defined as $1-\tau$

$\beta \quad$ see Eq. (14)

$\delta L \quad$ small path length increment (cm)

$\kappa_{\eta} \quad$ spectral absorption coefficient $\left(\mathrm{cm}^{-1}\right)$

$\Lambda \quad$ inverse of the second order $k$-moment absorptivity model, see Eq. (16) - (cm)

$\eta \quad$ wavenumber $\left(\mathrm{cm}^{-1}\right)$

$\tau \quad$ transmission function; transmissivity

\section{Subscripts}

$P \quad$ Planck mean

$R \quad$ Rosseland mean

$\langle n\rangle \quad$ related to the $k$-moment model at order $n$ 
$n \quad$ related to the $n$-th uniform sub-path along a non-uniform path

1..n related to the $L_{1}+L_{2}+. .+L_{n}$ non-uniform path

\title{
Superscripts
}

e effective

1,2 state 1 or 2 of the gas

$\Delta \eta \quad$ width of the spectral interval for the averaging of spectral properties

\section{Other notation}

$f \circ g$ represents the functional composition of functions $f$ and $g$ i.e. $f \circ g(x)=f[g(x)]$

\author{
Abbreviations \\ COG Curve-Of-Growth - see section 3 \\ EGA Emissivity Growth Approximation - Ref. [4] \\ GWN Godson-Weinreb-Neuendorffer's method - Ref. [7] \\ ICE Iterative Copula Evaluation - Ref. [1] \\ LBL Line-By-Line \\ LS-ICE Iterative Copula Evaluation with Levy Subordinators - this work \\ SNB Statistical Narrow Band model - Ref. [7]
}




\section{INTRODUCTION}

The recently proposed $\ell$-distribution method was introduced in Ref. [1] for the approximate modeling of the radiative properties of non-uniform gaseous media. It is built on the following ideas: 1/ the uniform $\ell$-distribution model is founded on a statistical method coupled with results taken from the $k$-moments method [1]. In practice, it mostly consists of a tabulation of a function that maps the LBL emissivities or absorptivities of uniform (homogeneous isothermal) gaseous paths averaged over spectral bands with an approximate model. The $\ell$-distribution method was shown in Ref. [1] to achieve LBL accuracy in uniform situations at very small computational expenses, $2 /$ an iterative scheme that propagates the radiation along a non-uniform layer in a stepby-step manner. This process is called the ICE (Iterative Copula Evaluation) scheme in the $\ell$ distribution method (it will be from now on referred to as ICE so as to abridge the notations). The principle of the technique to treat path non-uniformities is based on a scaling approximation similar to the one described by Godson in 1953 [2], almost at the same time as the widely used CurtisGodson approximation. Godson's technique was "rediscovered" in the early 70s by Weinreb and Neuendorffer [3] and slightly reformulated under the name EGA in Ref. [4]. The EGA technique has applications in radiative heat transfer in the atmosphere, and is used for instance in the codes JURASSIC [5] and BANDPAK [6]. In the recent book by Young [7], this method to propagate the radiation along a non-uniform path is called the "Godson-Weinreb-Neuendorffer's method" (GWN). The ICE scheme is similar to the GWN method in the sense that both techniques are based on the definition of effective scaling coefficients. However, this approximation is only one possibility to treat path non-uniformities in the $\ell$-distribution approach: a more general technique was described in Ref. [8]. 
The main problem with the ICE method is that it suffers from symmetry issue: this means that if one considers a non-uniform path represented as the juxtaposition of two uniform sub-paths, this technique does not ensure the same results to be obtained if the calculation of band averaged transmissivities is performed from right to left or from left to right. A similar problem of symmetry was noticed by Godson [2] in the frame of the Elssasser's model. One can observe that symmetry issues are not a particular feature of the ICE or GWN methods as comparable problems were reported with the Lindquist-Simmons approximation in Refs. [7,9].

The aim of the present paper is to propose a simple and accurate method to handle the symmetry issue encountered in the $\ell$-distribution / ICE method. The theoretical developments are extensively founded on the formulation used in the $\ell$-distribution approach: accordingly, and although some results provided here are general, understanding Ref. [1] is highly recommended to follow the ideas and mathematical derivations developed in this paper. The solution proposed to choose a direction of application of the non-uniform approximation inside a non-isothermal and non-homogeneous layer is shown to agree with the intuitive approaches described by Godson [2], Weinreb and Neuendorffer [3] and also discussed by Young [7] when restricted to Statistical Narrow Band formulations. The technique is assessed against Line-By-Line (LBL) calculations in situations representative of combustion applications (some of them are taken from Ref. [10]). This improved ICE scheme, called Levy Subordinated in order to employ the terminology used in Copula's theory that inspired the $\ell$-distribution method, is shown: $1 /$ to provide results as accurate as the $k$ distribution model, 2/ to improve the recently proposed ICE scheme for highly non-uniform applications. 


\section{PRINCIPLE OF THE ICE SCHEME}

The main idea behind the ICE scheme is the following one. Let us consider a gas in a given thermophysical state $\phi_{1}$. Small modifications of temperature, total pressure and species concentrations can be represented by a second state, $\phi_{2}$. If the changes between states $\phi_{1}$ and $\phi_{2}$ are small, the spectral absorption coefficients in the two states are similar but not rigorously the same (line intensities and profiles depend on the state of the gas). However, it is possible, as a first approximation, to estimate the band averaged transmissivities of a path of length $L$ in the gas in the state $\phi_{2}$ by the transmissivity of the gas in the state $\phi_{1}$. This requires defining an effective scaling coefficient between the spectra in the two states. More details are provided in Appendix A. This process then allows approximating transmissivities over non-uniform paths by transmissivities over equivalent isothermal and homogeneous layers. Fundamentally, these methods share many similarities with scaling approximations. Furthermore, one can notice that the ICE scheme was shown in Ref. [1] to coincide with the Curtis-Godson approximation at the optically thin and thick limits when applied to Statistical Narrow Band (SNB) models. A similar derivation was proposed in Ref. [11] for the GWN method.

More formally, let us consider a non-uniform layer discretized in two homogeneous isothermal sub-paths: the first one has a length $L_{1}$ and the gas is in the thermophysical state $\phi_{1}$, the second path has a length $L_{2}$ and the state of the gas is $\phi_{2}$. This is depicted in Figure 1. The spectral absorption coefficients in the two layers are $\kappa_{\eta}^{1}$ and $\kappa_{\eta}^{2}$ respectively. They are assumed strictly positive over a narrow band $\Delta \eta$ over which the Planck function is constant (the problem is thus formulated here within the frame of narrow band approaches). Our objective is to evaluate the 
transmissivity of the non-uniform path $L=L_{1}+L_{2}$ averaged over the band $\Delta \eta$. This transmissivity will be written $\tau_{12}^{\Delta \eta}\left(L_{1}, L_{2}\right)$. It is defined as:

$$
\tau_{12}^{\Delta \eta}\left(L_{1}, L_{2}\right)=\frac{1}{\Delta \eta} \int_{\Delta \eta} \exp \left(-\kappa_{\eta}^{1} L_{1}-\kappa_{\eta}^{2} L_{2}\right) d \eta
$$

As shown in Appendix A, the ICE scheme introduced in Ref. [1] provides an approximation of $\tau_{12}\left(L_{1}, L_{2}\right)$ as:

$$
\tau_{12}^{\Delta \eta}\left(L_{1}, L_{2}\right) \approx \tilde{\tau}_{12}^{\Delta \eta}\left(L_{1}, L_{2}\right)=\tilde{C}_{12}\left[\tau_{1}^{\Delta \eta}\left(L_{1}\right), \tau_{2}^{\Delta \eta}\left(L_{2}\right)\right]
$$

where:

$$
\tilde{C}_{12}\left(X_{1}, X_{2}\right)=\frac{1}{\Delta \eta} \int_{\Delta \eta} \exp \left(-\kappa_{\eta}^{2}\left[\ell_{2}\left(X_{1}\right)+\ell_{2}\left(X_{2}\right)\right]\right) d \eta
$$

Function $\ell_{2}(X), X \in[0,1]$ in Eq. (3) is defined as the inverse of the transmissivity $\tau_{2}^{\Delta \eta}\left(L_{2}\right)$ over the uniform path $L_{2}$, viz. $\ell_{2}\left[\tau_{2}^{\Delta \eta}\left(L_{2}\right)\right]=L_{2}$.

One can notice that the formulation used in Eq. (2) forces the thermophysical state $\phi_{2}$ to play a particular role. Indeed, the choice of this state to define function $\tilde{C}_{12}$ is quite arbitrary and Eq. (2) could have been written, a priori equivalently, as:

$$
\tau_{12}^{\Delta \eta}\left(L_{1}, L_{2}\right) \approx \tilde{\tau}_{21}^{\Delta \eta}\left(L_{1}, L_{2}\right)=\tilde{C}_{21}\left[\tau_{1}^{\Delta \eta}\left(L_{1}\right), \tau_{2}^{\Delta \eta}\left(L_{2}\right)\right]
$$

with:

$$
\tilde{C}_{21}\left(X_{1}, X_{2}\right)=\frac{1}{\Delta \eta} \int_{\Delta \eta} \exp \left(-\kappa_{\eta}^{1}\left[\ell_{1}\left(X_{1}\right)+\ell_{1}\left(X_{2}\right)\right]\right) d \eta
$$


This shows that there exist two possibilities to approximate the transmissivity of the non-uniform path. But these two equations take distinct mathematical forms and provide distinct results: this is depicted in Figure 2. The definition of criteria to choose between one or the other of these two sets of equations, Eqs. $(2,3)$ or Eqs. $(4,5)$, is thus required. This is the aim of the next section. 


\section{DEFINITION OF A MATHEMATICAL CRITERION TO HANDLE SYMMETRY}

ISSUES IN THE ICE SCHEME

The objective of the present section is to introduce a criterion that can be used to handle any kind of non-uniformity (of pressure and/or species concentrations and/or temperature). From now on, we will use the terminology "transmissivity / absorptivity" to represent "band averaged transmissivity / absorptivity" so as to abridge the notations.

For this purpose, let us start by reminding some mathematical properties of transmissivities of uniform gaseous paths. These transmissivities are completely monotonic (see Eq. (C.8) in Appendix C), which means that they are positive and their derivatives with respect to the length inside the gas $L$ alternate in sign: they are thus decreasing and concave up. This means that: $1 /$ the slope of a band averaged transmissivity curve, viz. $\partial \tau^{\Delta \eta}(L) / \partial L$, increases with respect to the path lengths, 2/ the transmissivity curve is above its tangent for any length $L$. This is shown schematically in Figure 3.

Notice that this property of transmissivities can also be seen as a consequence of the wellknown [7] concavity down property of the Curve Of Growth (COG) defined as $-\ln \tau^{\Delta \eta}(L)$. Indeed, narrow band COG are known to grow: 1/ quickly at the optically thin limit, where they are linear functions of the gas path length due to the fact that their rate of change is mostly governed by line centers, $2 /$ as the square root of the length of the gas path at the optically thick limit, for which their variations are mostly driven by the far wings of the lines. Accordingly, the rate of change of COG decreases with respect to the gas path length. They are thus concave down and transmissivities defined as $\exp (-\mathrm{COG})$ are then concave up. 
Let us now consider a non-uniform layer, as depicted in Figure 1. We define the ratio between the lengths of the two uniform gas path as $\rho=\frac{L_{2}}{L_{1}} \geq 0$.

The transmissivity of the non-uniform column $L=L_{1}+L_{2}$, given by Eq. (1), can be written in the same form as that of a uniform gas if we introduce an effective absorption coefficient:

$$
\kappa_{\eta, 12}^{e}=\frac{\kappa_{\eta}^{1}+\rho \cdot \kappa_{\eta}^{2}}{1+\rho}
$$

The corresponding band averaged transmission function, $\tau_{12}^{\Delta \eta, e}(L)$, has the same properties as that of a uniform path i.e. its is positive and decreasing. Moreover, its second derivative with respect to the total length of the path is positive:

$$
\frac{\partial^{2} \tau_{12}^{\Delta \eta, e}(L)}{\partial L^{2}}=\frac{1}{\Delta \eta} \int_{\Delta \eta}\left[\kappa_{\eta, 12}^{e}\right]^{2} \exp \left(-\kappa_{\eta, 12}^{e} L\right) d \eta \geq 0
$$

Let us increase the length of the layer inside the gas with uniform absorption coefficient $\kappa_{\eta, 12}^{e}$ by a small quantity $\delta L$. This provides the same value of transmissivity as if we modify the lengths of the original non-uniform path $L=L_{1}+L_{2}$ by adding $\delta L_{1}=\delta L /(1+\rho)$ to the column on the right in Figure 1 and $\delta L_{2}=\rho \cdot \delta L /(1+\rho)$ to the second layer. This can be written as:

$$
\tau_{12}^{\Delta \eta, e}(L+\delta L)=\tau_{12}^{\Delta \eta}\left[L_{1}+\delta L /(1+\rho), L_{2}+\rho \cdot \delta L /(1+\rho)\right]
$$

The Taylor-Lagrange expansion of Eq. (8) with respect to $\delta L$ provides, after identification of the terms at the second order:

$$
\frac{\partial^{2} \tau_{12}^{\Delta \eta, e}(L)}{\partial L^{2}}=\frac{1}{(1+\rho)^{2}} \frac{\partial^{2} \tau_{12}^{\Delta \eta}\left(L_{1}, L_{2}\right)}{\partial\left\{L_{1}\right\}^{2}}+\frac{\rho^{2}}{(1+\rho)^{2}} \frac{\partial^{2} \tau_{12}^{\Delta \eta}\left(L_{1}, L_{2}\right)}{\partial\left\{L_{2}\right\}^{2}}+\frac{2 \rho}{(1+\rho)^{2}} \frac{\partial^{2} \tau_{12}^{\Delta \eta}\left(L_{1}, L_{2}\right)}{\partial L_{1} \partial L_{2}}
$$


It can be checked without difficulty that the sum of the three terms at the RHS in Eq. (9) complies with the inequality Eq. (7). The same expansion can be evaluated if one uses the approximation set by Eqs. (2,3). This yields the following estimate for the RHS in Eq. (9):

$$
\frac{1}{(1+\rho)^{2}} \frac{\partial^{2} \tilde{\tau}_{12}^{\Delta \eta}\left(L_{1}, L_{2}\right)}{\partial\left\{L_{1}\right\}^{2}}+\frac{\rho^{2}}{(1+\rho)^{2}} \frac{\partial^{2} \tilde{\tau}_{12}^{\Delta \eta}\left(L_{1}, L_{2}\right)}{\partial\left\{L_{2}\right\}^{2}}+\frac{2 \rho}{(1+\rho)^{2}} \frac{\partial^{2} \tilde{\tau}_{12}^{\Delta \eta}\left(L_{1}, L_{2}\right)}{\partial L_{1} \partial L_{2}}
$$

where:

$$
\begin{aligned}
& \frac{\partial^{2} \tilde{\tau}_{12}^{\Delta \eta}\left(L_{1}, L_{2}\right)}{\partial\left\{L_{1}\right\}^{2}}=\underbrace{\left[\frac{\partial \ell_{2} \circ \tau_{1}^{\Delta \eta}\left(L_{1}\right)}{\partial L_{1}}\right]^{2} \cdot \frac{1}{\Delta \eta} \int_{\Delta \eta}\left(\kappa_{\eta}^{2}\right)^{2} \exp \left(-\kappa_{\eta}^{2} \cdot\left[\ell_{2} \circ \tau_{1}^{\Delta \eta}\left(L_{1}\right)+L_{2}\right]\right) d \eta}_{\geq 0} \\
& -\frac{\partial^{2} \ell_{2} \circ \tau_{1}^{\Delta \eta}\left(L_{1}\right)}{\partial\left\{L_{1}\right\}^{2}} \cdot \underbrace{\frac{1}{\Delta \eta} \int_{\Delta \eta} \kappa_{\eta}^{2} \exp \left(-\kappa_{\eta}^{2} \cdot\left[\ell_{2} \circ \tau_{1}^{\Delta \eta}\left(L_{1}\right)+L_{2}\right]\right) d \eta}_{\geq 0} \\
& \frac{\partial^{2} \tilde{\tau}_{12}^{\Delta \eta}\left(L_{1}, L_{2}\right)}{\partial\left\{L_{2}\right\}^{2}}=\underbrace{\frac{1}{\Delta \eta} \int_{\Delta \eta}\left(\kappa_{\eta}^{2}\right)^{2} \exp \left(-\kappa_{\eta}^{2} \cdot\left[\ell_{2} \circ \tau_{1}^{\Delta \eta}\left(L_{1}\right)+L_{2}\right]\right) d \eta}_{\geq 0} \\
& \frac{\partial^{2} \tilde{\tau}_{12}^{\Delta \eta}\left(L_{1}, L_{2}\right)}{\partial L_{1} \partial L_{2}}=\underbrace{\frac{\partial \ell_{2} \circ \tau_{1}^{\Delta \eta}\left(L_{1}\right)}{\partial L_{1}} \cdot \frac{1}{\Delta \eta} \int_{\Delta \eta}\left(\kappa_{\eta}^{2}\right)^{2} \exp \left(-\kappa_{\eta}^{2} \cdot\left[\ell_{2} \circ \tau_{1}^{\Delta \eta}\left(L_{1}\right)+L_{2}\right]\right) d \eta}_{\geq 0}
\end{aligned}
$$

In order to be physically realistic, the non-uniform approximation provided by Eqs. $(2,3)$ needs to ensure the quantity given by Eq. (10) to be positive, so as to comply with the constraint Eq. (7). Following Eqs. (11), a sufficient condition to ensure the validity of Eq. (7) for any couple of lengths $L_{1}$ and $L_{2}$ is thus:

$$
\frac{\partial^{2} \ell_{2} \circ \tau_{1}^{\Delta \eta}\left(L_{1}\right)}{\partial\left\{L_{1}\right\}^{2}} \leq 0, L_{1} \in[0,+\infty)
$$


If the condition (12) is not met, it is almost impossible to conclude (at least in a simple way) if the non-uniform model is in accordance or not with the constraint Eq. (7). However, in this case, choosing Eqs. $(4,5)$ to treat the non-uniform path instead of Eqs. $(2,3)$ ensures $(7)$ to be correct, as shown below: it is then a more reasonable choice.

Indeed, let us prove that this criterion is discriminant viz. that, given two states, $\ell_{1} \circ \tau_{2}^{\Delta \eta}$ and $\ell_{2} \circ \tau_{1}^{\Delta \eta}$ cannot satisfy Eq. (12) simultaneously. For this purpose, we first notice that $\ell_{1} \circ \tau_{2}^{\Delta \eta}$ is the inverse of $\ell_{2} \circ \tau_{1}^{\Delta \eta}$ and that both functions are strictly increasing. Furthermore, the sign of their second derivative with respect to the gas path length can be reasonably considered to remain the same for any length, as will be shown later in section 4 (see Eq. (20)). Two situations need to be considered:

- If for instance $\frac{\partial^{2} \ell_{2} \circ \tau_{1}^{\Delta \eta}\left(L_{1}\right)}{\partial\left\{L_{1}\right\}^{2}}<0$, then function $\ell_{2} \circ \tau_{1}^{\Delta \eta}$ is concave down (its first derivative decreases with respect to $L_{1}$ ). Its inverse $\ell_{1} \circ \tau_{2}^{\Delta \eta}$ is thus concave up, which shows that $\frac{\partial^{2} \ell_{1} \circ \tau_{2}^{\Delta \eta}\left(L_{2}\right)}{\partial\left\{L_{2}\right\}^{2}}>0$ (this quantity appears in the formulation of Eq. (10) obtained by using Eqs. $(5,6)$ instead of Eqs. $(2,3))$. This means that the second derivatives of $\ell_{1} \circ \tau_{2}$ and $\ell_{2} \circ \tau_{1}$ cannot share the same sign: if one of them is strictly positive, the other one is automatically negative.

- If $\frac{\partial^{2} \ell_{2} \circ \tau_{1}^{\Delta \eta}\left(L_{1}\right)}{\partial\left\{L_{1}\right\}^{2}}=0$ then $\ell_{2} \circ \tau_{1}^{\Delta \eta}$ is linear, viz. $\ell_{2} \circ \tau_{1}^{\Delta \eta}\left(L_{1}\right)=a+b L_{1}$. The constant coefficient is obviously null, since $\ell_{2} \circ \tau_{1}^{\Delta \eta}(0)=a=0$. In this case, gas spectra are scaled (the ratio of the two spectra is a constant) and the scaling coefficient is simply the slope of 
the linear function, viz. for any $\eta \in \Delta \eta, \kappa_{\eta}^{1}=b \cdot \kappa_{\eta}^{2}$. In this particular case, the calculation can be done in any direction, as it provides exactly the same result.

The condition Eq. (12) can thus be used as a decisive factor to choose a direction for the calculation of the transmissivities of the non-uniform path. It can be applied to any band model and can be readily extended over the full spectrum with minor modifications. Furthermore, it can be also reformulated in a simple manner if one uses the formalism introduced in the narrow band $\ell$ distribution approach. This is detailed in the next section. 


\section{REFORMULATION OF THE CRITERION SET BY EQ. (12) WITHIN THE FRAME OF THE NARROW BAND -DISTRIBUTION APPROACH}

In the $\ell$-distribution method, as described in Ref. [1], one starts by defining a "germ" absorptivity model whose most essential feature is to have an analytical inverse. It was shown in Ref. [1] that any model obtained by application of the $k$-moments method $[7,12]$ can be used for this purpose. The second order $k$-moments approximation, which shares the same mathematical form as the SNB model for Lorentz lines with Malkmus' distribution of linestrengths, was shown in the same reference to be a relevant candidate. The "germ" absorptivity function is in this case (the index inside the brackets corresponds here to the second order $k$-moments model, not to the second thermophysical state):

$$
\alpha_{\langle 2\rangle}^{\Delta \eta}(L)=1-\exp \left[-\frac{\beta}{\pi}\left(\sqrt{1+\frac{2 \pi k_{P} L}{\beta}}-1\right)\right]
$$

In Eq. (13), the coefficient:

$$
\beta=\pi\left(\frac{k_{P}}{k_{R}}-1\right)^{-1}
$$

plays the role of an overlapping parameter and:

$$
\left\{\begin{array}{l}
k_{P}=\frac{1}{\Delta \eta} \int_{\Delta \eta} \kappa_{\eta} d \eta \\
k_{R}^{-1}=\frac{1}{\Delta \eta} \int_{\Delta \eta} \frac{d \eta}{\kappa_{\eta}}
\end{array}\right.
$$

are the Planck (index $P$ ) and Rosseland (index $R$ ) mean absorption coefficients of the gas over the narrow band. 
It can be noticed that if the gas spectrum follows rigorously the assumptions of the SNB model for Lorentz lines with Malkmus' distribution of linestrengths, then the coefficient $\beta$ given by Eq. (14) matches exactly the overlapping parameter encountered in this model.

The inverse of Eq. (13) is analytical and is given as:

$$
\Lambda(X)=\frac{\beta}{2 \pi k_{P}} \cdot\left[\left(1-\frac{\pi}{\beta} \ln (1-X)\right)^{2}-1\right]
$$

The second step in the building of the $\ell$-distribution model is to define a function, called a rank transmutation map following the terminology proposed in Ref. [13], that maps the [0,1] interval into itself and that associates the values taken by the "germ" model at a given length $L$ with those obtained by the LBL approach. This function is simply defined as [1]:

$$
G r(X)=1-\frac{1}{\Delta \eta} \int_{\Delta \eta} \exp \left[-\kappa_{\eta} \cdot \Lambda(X)\right] d \eta, X \in[0,1]
$$

Values of the $G r$ function can be readily evaluated by application of Eq. (17) to LBL data and stored in tables. It can be noticed that if the gas spectrum follows rigorously the assumptions of the SNB model for Lorentz lines with the Malkmus' distribution of linestrengths, then $G r(X)=X$.

The approximate model used in the $\ell$-distribution approach to evaluate absorptivities of uniform paths involves a two step process, for a given path length $L: 1 /$ evaluate the value of the germ model at the value $L, 2 /$ map this value to the "true" one using the $G r$ function. This involves an interpolation of the values of $G r$ stored in the table. The absorptivity is thus estimated in the $\ell-$ distribution approach as $\alpha^{\Delta \eta}(L)=G r\left[\alpha_{\langle 2\rangle}^{\Delta \eta}(L)\right]$. 
This formulation also provides an explicit formula for the function $\ell_{2} \circ \tau_{1}^{\Delta \eta}$ (see Ref. [1] - Eq. (18) below is analogous to Eq. (32) from this reference):

$$
\ell_{2} \circ \tau_{1}^{\Delta \eta}\left(L_{1}\right)=\Lambda_{2} \circ\left(G r_{2}^{-1} \circ G r_{1}\right) \circ \alpha_{\langle 2\rangle, 1}^{\Delta \eta}\left(L_{1}\right)
$$

in which the indices without brackets refer to the thermophysical states of the gas.

Now, if one considers two close thermophysical conditions (which is the main assumption used to derive the ICE scheme, as shown in Appendix A, and theoretically restricts its range of validity), then one can reasonably assume, as a first approximation, that the two mapping functions are the same viz. $G r_{2}^{-1} \circ G r_{1}(X) \approx X, X \in[0,1]$. This is due to the fact that $G r$ functions are smooth, and depend weakly on the thermophysical states of the gas (see for instance Figure 1, from Ref. [1]). This allows writing Eq. (18) as:

$$
\ell_{2} \circ \tau_{1}^{\Delta \eta}\left(L_{1}\right)=\frac{k_{P, 1}}{k_{P, 2}} \frac{\beta_{1}}{\beta_{2}} L_{1}+\frac{k_{P, 1}}{k_{P, 2}}\left(1-\frac{\beta_{1}}{\beta_{2}}\right) \frac{\beta_{1}}{\pi k_{P, 1}}\left(\sqrt{1+\frac{2 \pi k_{P, 1} L_{1}}{\beta_{1}}}-1\right)
$$

The condition set by Eq. (12) requires evaluating the second derivative of the previous function Eq. (19) with respect to $L_{1}$ :

$$
\frac{\partial^{2} \ell_{2} \circ \tau_{1}^{\Delta \eta}\left(L_{1}\right)}{\partial\left\{L_{1}\right\}^{2}}=-\frac{\pi k_{P, 1}}{\beta_{1}} \frac{k_{P, 1}}{k_{P, 2}}\left(1-\frac{\beta_{1}}{\beta_{2}}\right)\left(1+\frac{2 \pi k_{P, 1} L_{1}}{\beta_{1}}\right)^{-3 / 2}
$$

This quantity is thus negative or null if and only if $1-\frac{\beta_{1}}{\beta_{2}} \geq 0$ or equivalently, using Eq. (14), as soon as $\frac{k_{P, 1}}{k_{R, 1}} \geq \frac{k_{P, 2}}{k_{R, 2}}$. If this condition over the parameters $\frac{k_{P, i}}{k_{R, i}}, i=1,2$ is fulfilled, then the criterion set by Eq. (12) is verified. Further comments about the physical meaning of this criterion are provided in Appendix B. 
An advantage of the formulation of the criterion Eq. (12) in terms of $\frac{k_{P}}{k_{R}}$ is that it simplifies its practical implementation, as it only requires reordering the non-uniform layers with respect to decreasing values of the parameters $\frac{k_{P}}{k_{R}}$ (which are quantities required for the calculation of the transmissivities). It can be noticed that this criterion over the parameter $\frac{k_{P}}{k_{R}}$ complies with earlier intuitive methods proposed by: 1/ Godson, who founded his analysis on the overlapping parameter which is, in the case of the Elsasser's model used in Ref. [2], directly related to $\frac{k_{P}}{k_{R}}$. More details about this technique are given in Section 5, 2/ Weinreb and Neuendorffer, who suggested applying the method in the direction of increasing absorption rates, which corresponds to the direction of decreasing $\frac{k_{P}}{k_{R}}$ values, 3/ Young who reached the same conclusion by using a band model based on Malkmus' distribution function of linestrengths and compared the results with reference calculations obtained by application of Rodger's two-cell approximation (see Section 12.4 from Ref. [7]). This reformulation of the condition set by Eq. (12) into a simple and readily applicable form is totally founded on the formulation used in the $\ell$-distribution approach.

Notice also that the condition $\frac{k_{P, 1}}{k_{R, 1}} \geq \frac{k_{P, 2}}{k_{R, 2}}$ does not only ensure $\frac{\partial \ell_{2} \circ \tau_{1}^{\Delta \eta}\left(L_{1}\right)}{\partial L_{1}}$ to have a negative derivative, but it implies all the derivatives to alternate in sign. In this case, $\ell_{2} \circ \tau_{1}^{\Delta \eta}\left(L_{1}\right)$ takes the form of a Levy subordinator. This terminology, taken from copula's theory, will be used to refer to this modified ICE scheme as Levy Subordinated in the following. More details about copulas and more precisely about Levy Subordinated Archimedean copulas are provided in Appendix C. 
The Levy Subordinated ICE (LS-ICE) scheme mostly consists in an application of the ICE method over paths reordered with respect to decreasing values of the parameter $\frac{k_{P}}{k_{R}}$. The principles of ICE and LS-ICE methods are depicted in Figures 4. These schemes are evaluated in the next section against reference LBL calculations in non-uniform situations representative of radiative heat transfer in flames. 


\section{APPLICATIONS}

\subsection{Model parameters}

The $\ell$-distribution model parameters used in the present work are the same as those described in Ref. [1]. The interested readers should refer to this paper for additional details. The C-k model is also the same as in Ref. [1]: it uses 16 values of absorption coefficient and the integration over pseudo-wavenumbers (the cumulative $k$-distribution) is based on a Gauss-Legendre quadrature. The subroutine used for the reordering of the gas path with respect to the values taken by the parameters $\frac{k_{P}}{k_{R}}$ in the LS-ICE method was taken from Ref. [14].

\subsection{Assessment of model's predictions against reference LBL calculations}

The first two cases $\left(\mathrm{C} 1\right.$ and $\mathrm{C} 2$ ) concern non-uniform gaseous mixtures of $\mathrm{CO}_{2}$ or $\mathrm{H}_{2} \mathrm{O}$ with $\mathrm{N}_{2}$. They were adapted from Ref. [9]. They consist of 3 uniform layers at different temperatures with the same amount of emitting species $x P L=5.0 \mathrm{~cm}$.atm in all columns: the parameters for the columns are (with the notation: temperature / species concentration of absorbing gas / length) 1500 $\mathrm{K} / 0.5 / 10 \mathrm{~cm}$ for the first column, $1000 \mathrm{~K} / 0.2$ / $25 \mathrm{~cm}$ for the second layer and $500 \mathrm{~K} / 0.05$ / $100 \mathrm{~cm}$ for the third one. In these two cases, the path is ordered in such a way that the ICE method is applied in the direction of increasing $\frac{k_{P}}{k_{R}}$ parameters (the hot column is thus close to the exit of the path). Results of Godson's method - that was evaluated by averaging the transmissivities of the non-uniform path in the directions of increasing and decreasing $\frac{k_{P}}{k_{R}}$ - are also provided. These transmissivities, noted "Godson's method", were thus calculated as the average between the 
transmissivity obtained with the ICE technique and the transmissivity provided by application of the LS-ICE method.

The first case (C1, Figure 5) corresponds to calculations performed over the $2.7 \mu \mathrm{m}$ band of $\mathrm{CO}_{2}$ and the second one (C2, Figure 6$)$ to water vapor over the $[1000 ; 4500] \mathrm{cm}^{-1}$ spectral interval. The mean relative errors evaluated over the bands shown in Figure 5 and Figure 6 is one order of magnitude lower for the LS-ICE model (-0.0007 for Case C1, -0.0003 for case C2) than for the C$k$ model (-0.007 for both Cases C1 and C2). Godson's method provides values similar to the C- $k$ approach; the ICE method lead to higher values of mean relative errors, around - 0.01 for Cases C12. Results provided by the LS-ICE method are clearly more accurate in these situations than both the ICE and C- $k$ methods. It is worth noticing that Godson's intuitive approach is less accurate than the LS-ICE scheme but provides results which are very close to those obtained with the C- $k$ model. Cases C3-5 were taken from Ref. [10]. They consist of four uniform paths (mixtures of $\mathrm{H}_{2} \mathrm{O}$ and $\mathrm{N}_{2}$ ) in series and are representative of flame emission spectra. The non-uniform optical path parameters used for the computation of the emitted band average intensity (the radiation propagates from layer 4 to 1 ) are given in Table 1. Comparisons with reference LBL calculations are depicted in Figures 7-9. In these cases, the accuracy of the LS-ICE method is very high, similar or superior to the C- $k$ model. In case $\mathrm{C} 4$, the ICE and LS-ICE methods provide exactly the same results: this is due to the fact that this case corresponds to a situation for which the non-uniform path is already reordered in the direction of decreasing $\frac{k_{P}}{k_{R}}$ parameters. In terms of total band averaged intensity, the best results are in most cases obtained with the LS-ICE method. This is detailed in Table 2.

Finally, Case C6 corresponds to the same profile of temperature and species concentrations as used in Ref. [10] (see Case 1 from this reference). The optical path was divided in 9 isothermal layers 
with the same length $6.65 \mathrm{~cm}$. Values of temperature used in the calculation were obtained at the centers of the layers, by interpolating the values provided in the caption to Figure 7 from Ref. [10] viz. 459.5 K, 630.0 K, 838.0 K, 1040.0 K, 1144.0 K, 1075.0 K, 870.5 K, 654.5 K, and 473.5 K. Pure $\mathrm{H}_{2} \mathrm{O}$ is considered and no pressure or species concentration gradients are encountered along the non-uniform path. Results are depicted in Figure 10.

\subsection{Discussion}

From the results described above, the following comments can be drawn:

- The LS-ICE method usually provides results more accurate than the ICE technique when large gradients of temperature are encountered along the optical path. When the uniform layers are already ordered in the direction of decreasing $\frac{k_{P}}{k_{R}}$ parameters (this is most often the case when the path is treated from the cold to the hot regions), the spectra predicted by the two ICE schemes are rigorously the same.

- Godson's method provides more accurate results than the ICE technique but also less precise than the LS-ICE method. Results of this technique are close to those obtained by the C- $k$ model.

- Results provided by the LS-ICE and C- $k$ models are similarly accurate. However, the LSICE technique appears to be slightly more precise than C- $k$ for band averaged calculations (see Table 2).

One of the main defects of the LS-ICE method is that it requires a reordering of the non-uniform path with respect to decreasing values of the $\frac{k_{P}}{k_{R}}$ parameters. This raises the computational cost of the method compared to a "basic" ICE scheme. This limitation is not really problematical 
for the calculation of transmissivities over non-uniform paths but increases significantly the computational cost of the method for intensity calculations: this is due to the fact that, in this case, any non-uniform layer needs to be reordered and the calculation of non-uniform sub-paths transmissivities needs to be done completely, viz. by restarting the calculation of all of them from the beginning of the path.

For this reason, the LS-ICE scheme cannot be recommended as a general purpose gas radiation model for highly non-isothermal media but the $\ell$-distribution method was not designed for this purpose, as explicitly written in Ref. [1]. However, this method appears to be a relevant candidate for applications that require a high accuracy, as is the case for instance in flame or atmospheric spectroscopy analysis. 


\section{CONCLUSION}

The aim of the present paper was threefold: $1 /$ to investigate the reasons that may explain the symmetry issue encountered in the ICE method; $2 /$ to propose a simple criterion to handle this problem for radiative heat transfer calculations; 3 / to assess the accuracy of the proposed decisive factor in situations representative of radiative heat transfer in highly non-uniform gaseous media.

We have shown that it is possible to choose a direction of propagation for the ICE scheme that ensures the transmissivity of the non-uniform path to be physically realistic viz. concave up. The proposed criterion was reformulated in terms of the ratio $\frac{k_{P}}{k_{R}}$. This reformulation allows its simple implementation for radiative transfer calculation and also its possible use together with a wide range of possible techniques, including narrow band and full spectrum $k$-distribution models. This criterion agrees with the intuitive approaches proposed by Godson in 1953 and Weinreb and Neuendorffer in the early 70s when applied to the Statistical Narrow Band model with the Malkmus' distribution function of linestrengths. Comparisons of the proposed modifications of the ICE scheme were shown to improve significantly the model accuracy for radiative heat transfer in highly non-isothermal gases. Once combined with the "usual" l-distribution approach, this scheme provides in most cases more accurate results than the C- $k$ model. The $\ell$-distribution / LS-ICE method appears to be a relevant candidate for applications that necessitate a high accuracy at a reasonable computational cost, such as required for instance in quantitative flame / atmospheric spectroscopy. 


\section{AKNOWLEDGEMENTS}

This work has been supported by the Programme National de Télédétection Spatiale (PNTS, http://www.insu.cnrs.fr/pnts grant $N^{\circ}$ PNTS-2016-05). 


\section{REFERENCES}

[1] ANDRE F. The $\ell$-distribution method for modeling non-gray absorption in uniform and nonuniform gaseous media, JQSRT 2016;179:19-32.

[2] GODSON W.L. The evaluation of infra-red radiative fluxes due to atmospheric water vapor, Q. J. R. Met. Soc. 1953;79:367-379.

[3] WEINREB M.P., NEUENDORFFER A.C. Method to apply homogeneous-path transmittance models to inhomogeneous atmospheres, J. Atm. Sci. 1973;30:662-666.

[4] GORDLEY L.L., RUSSELL III J.M. Rapid inversion of limb radiance data using an emissivity growth approximation, App. Opt. 1981;20:807-813.

[5] GRIESSBACH S., HOFFMANN L., HÖPFNER M., RIESE M., SPANG R. Scattering in infrared radiative transfer: A comparison between the spectrally averaging model JURASSIC and the line-by-line model KOPRA, JQSRT 2013;127:102-118.

[6] MARSHALL B.T., GORDLEY L.L., CHU A.D. BANDPAK: Algorithms for modeling broadband transmission and radiance, JQSRT 1994;52:581-599.

[7] YOUNG S.J. Band model theory of radiation transport, The Aerospace Press, 2013. ISBN: 978$1-884989-25-4$.

[8] ANDRE F. A polynomial chaos approach to narrow band modeling of radiative heat transfer in non-uniform gaseous media, JQSRT 2016;175:17-29.

[9] SOUFIANI A., HARTMANN J.M., TAINE J. Validity of band model calculations for $\mathrm{CO}_{2}$ and $\mathrm{H}_{2} \mathrm{O}$ applied to radiative properties and conductive-radiative transfer, JQSRT 1985;33:243-257.

[10] YOUNG S.J. Evaluation of nonisothermal band models for $\mathrm{H}_{2} \mathrm{O}$, JQSRT 1977;18:29-45. 
[11] PIERLUISSI J. H., RUFUS E. B. Discussion on equivalence methods for inhomogoneous paths, J. Atm. Sci. 1975;32:1497-1499.

[12] ANDRE F., VAILLON R. The $k$-moment method for modeling the blackbody weighted transmission function for narrow and wide band radiative properties of gases, JQSRT 2007;108:116.

[13] SHAW W.T., BUCKLEY I.R.C. The alchemy of probabilistic distributions: behond GramCharlier expansions, and a skew-kurtotic-normal distribution from a rank transmutation map, presented at the First IMA Computational Finance Conference, $23^{\text {rd }}$ March 2007.

[14] Numerical recipes in Fortran 77: the art of scientific computing. Cambridge: Cambridge University Press; 1992.

[15] NELSEN R.B. An introduction to Copulas - Second Edition, Springer series in statistics, Springer, 2006.

[16] BALAKRISHNAN N., LAI C-D. Continuous bivariate distributions - Second edition, Springer, 2009.

[17] JOE H. Multivariate models and dependence concepts, Monograph on statistics and applied probability 73, Springer Science+Business Media Dordrecht 1997.

[18] SKLAR A. Fonctions de répartitions et leurs marges, Publications of the Institute of Statistics, Université de Paris, 1959;8:229-231.

[19] McNEIL A.J., NESLEHOVA J. Multivariate Archimedean copulas, $d$-monotone functions and $\ell_{1}$-norm symmetric distributions, The Annals of Statistics 2009;37:3059-3097. 
[20] HERING C., HOFERT M., MAI J.-F., SCHERE M., Constructing hierarchical Archimedean copulas with Levy subordinators, J. Multivariate Analysis 2010;101:1428-1433.

[21] ANDRE F., VAILLON R. Generalization of the $k$-moment method using the maximum entropy principle. Application to the NBKM and full spectrum SLMB gas radiation models, JQSRT 2012;113:1508-1520. 


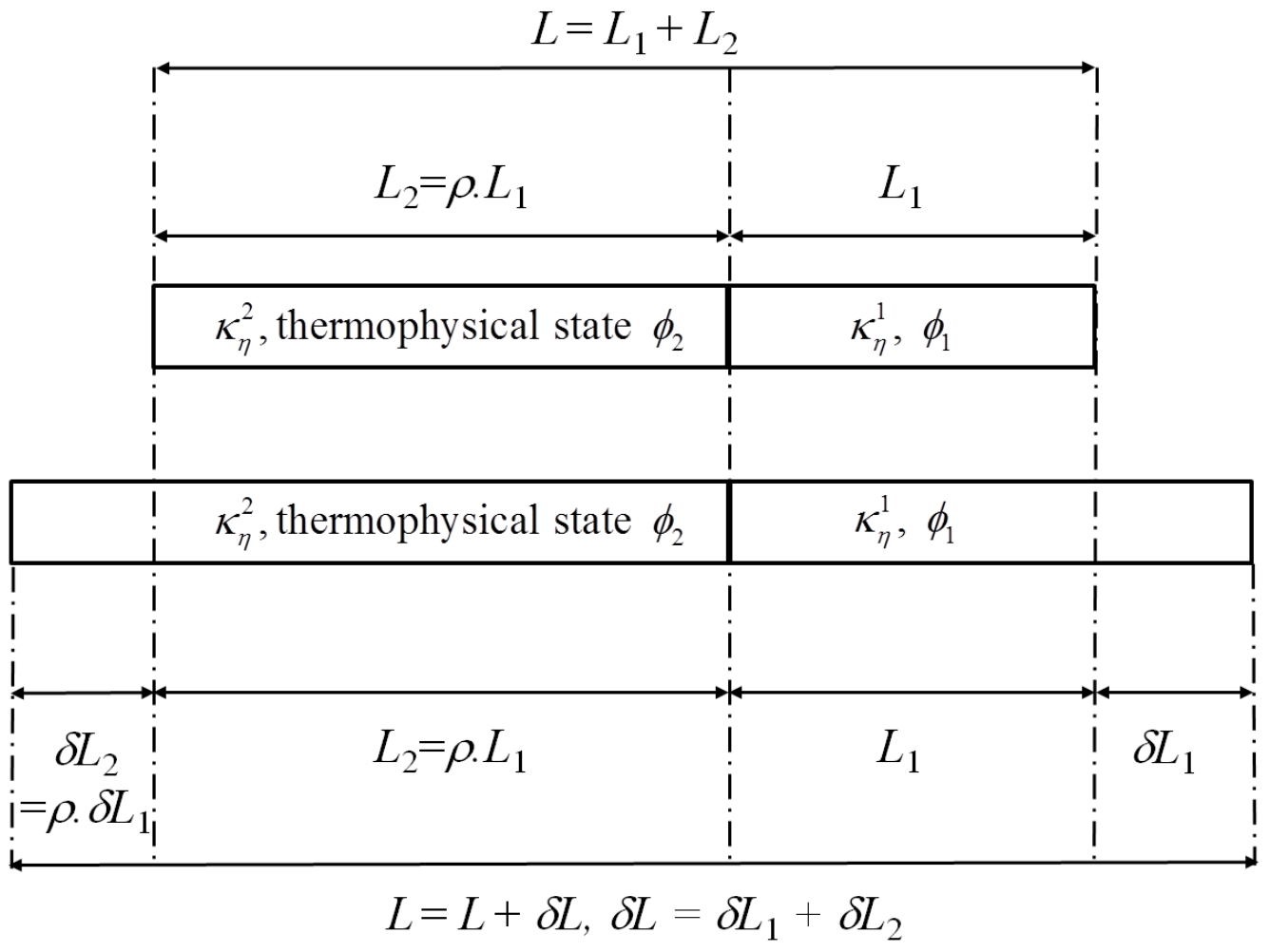

Figure 1. Schematic representation of a non-uniform gaseous path. 


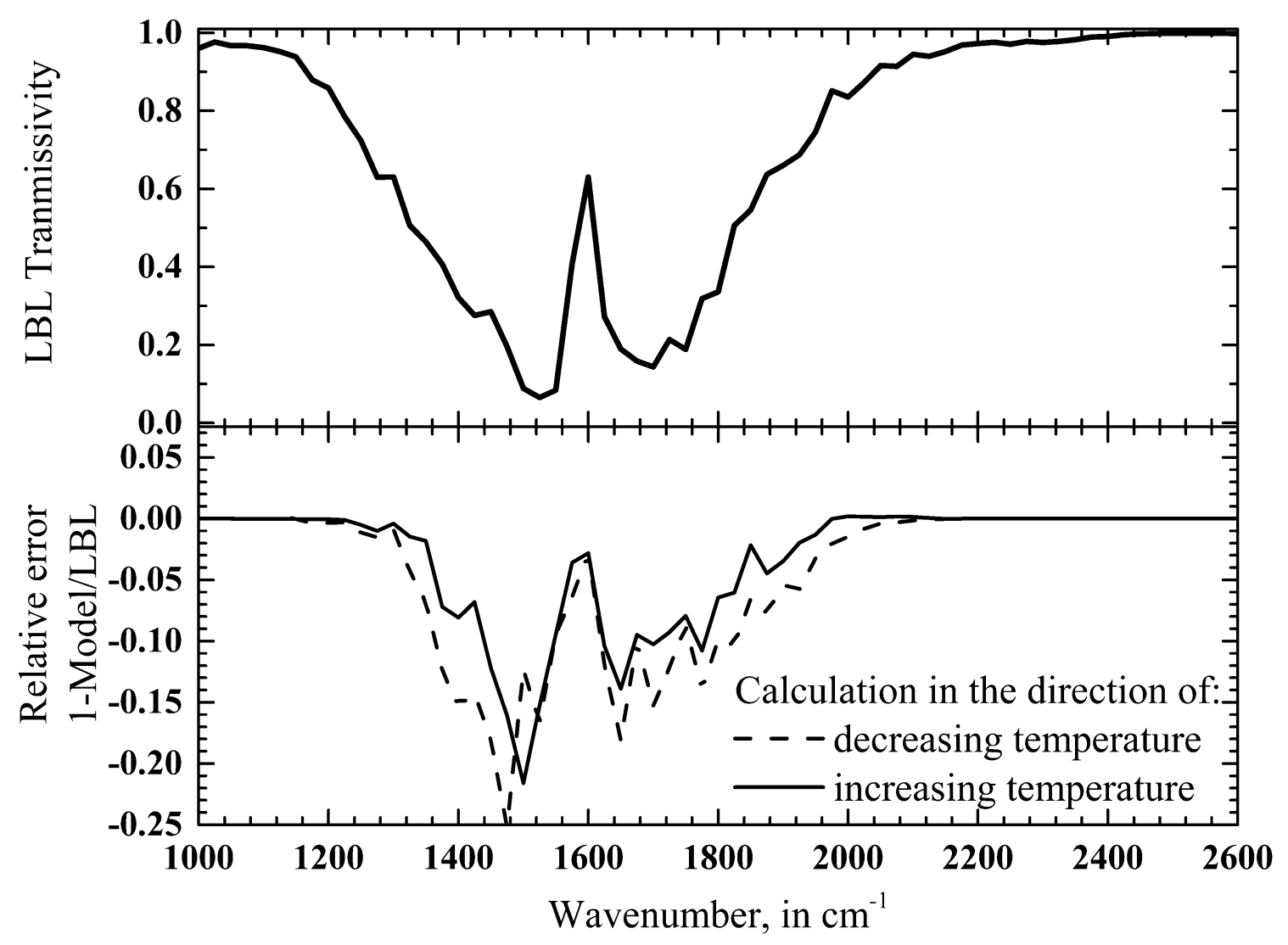

Figure 2. Illustration of the asymmetry issue in the ICE scheme. The cold layer is at $300 \mathrm{~K}$, the hot one at $1000 \mathrm{~K} . \mathrm{H}_{2} \mathrm{O}(20 \%)-\mathrm{N}_{2}(80 \%)$ mixture at atmospheric pressure. The length of both layers is $50 \mathrm{~cm}$. 
Transmissivity, $\tau^{\Delta \eta}$

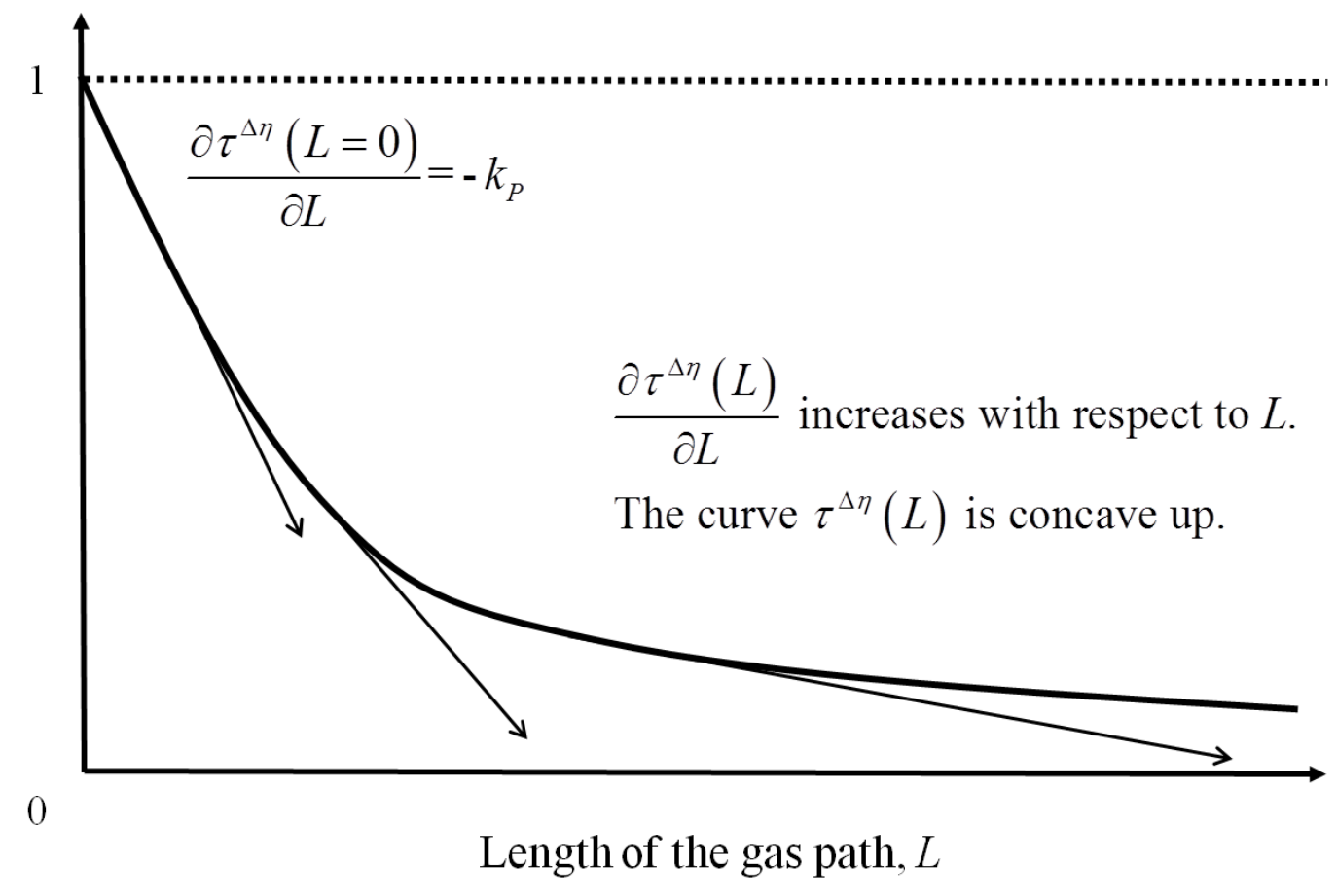

Figure 3. Anatomy and properties of narrow band transmissivity curves. 


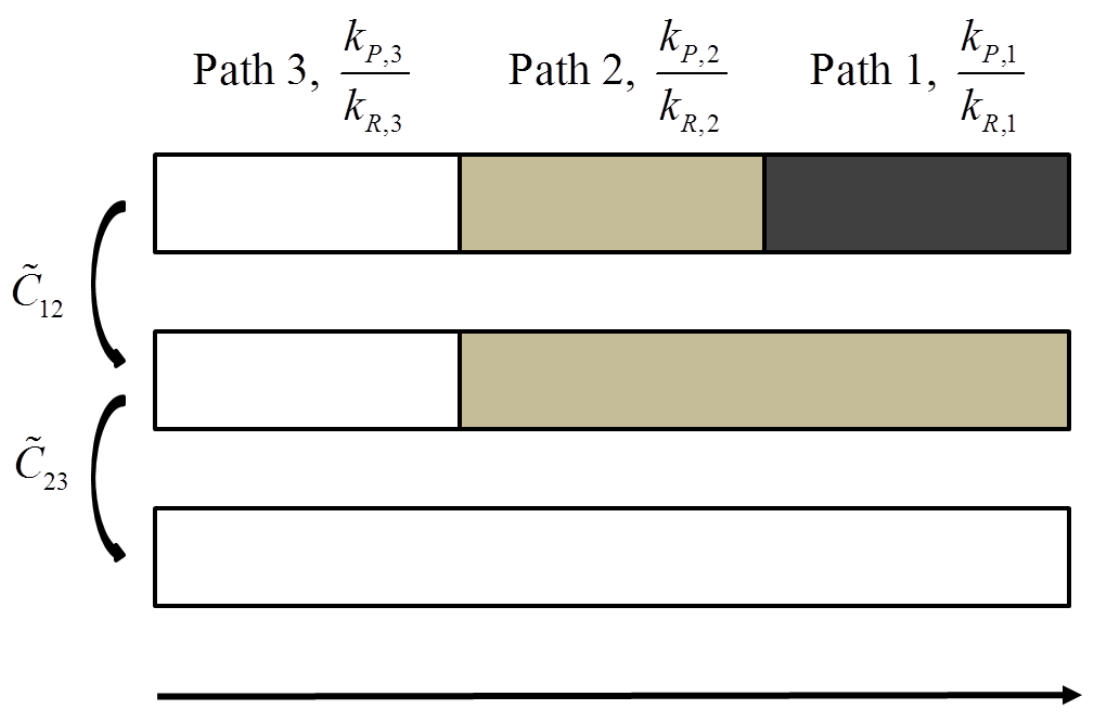

Figure 4-a. ICE scheme. Radiation propagates from left to right. 


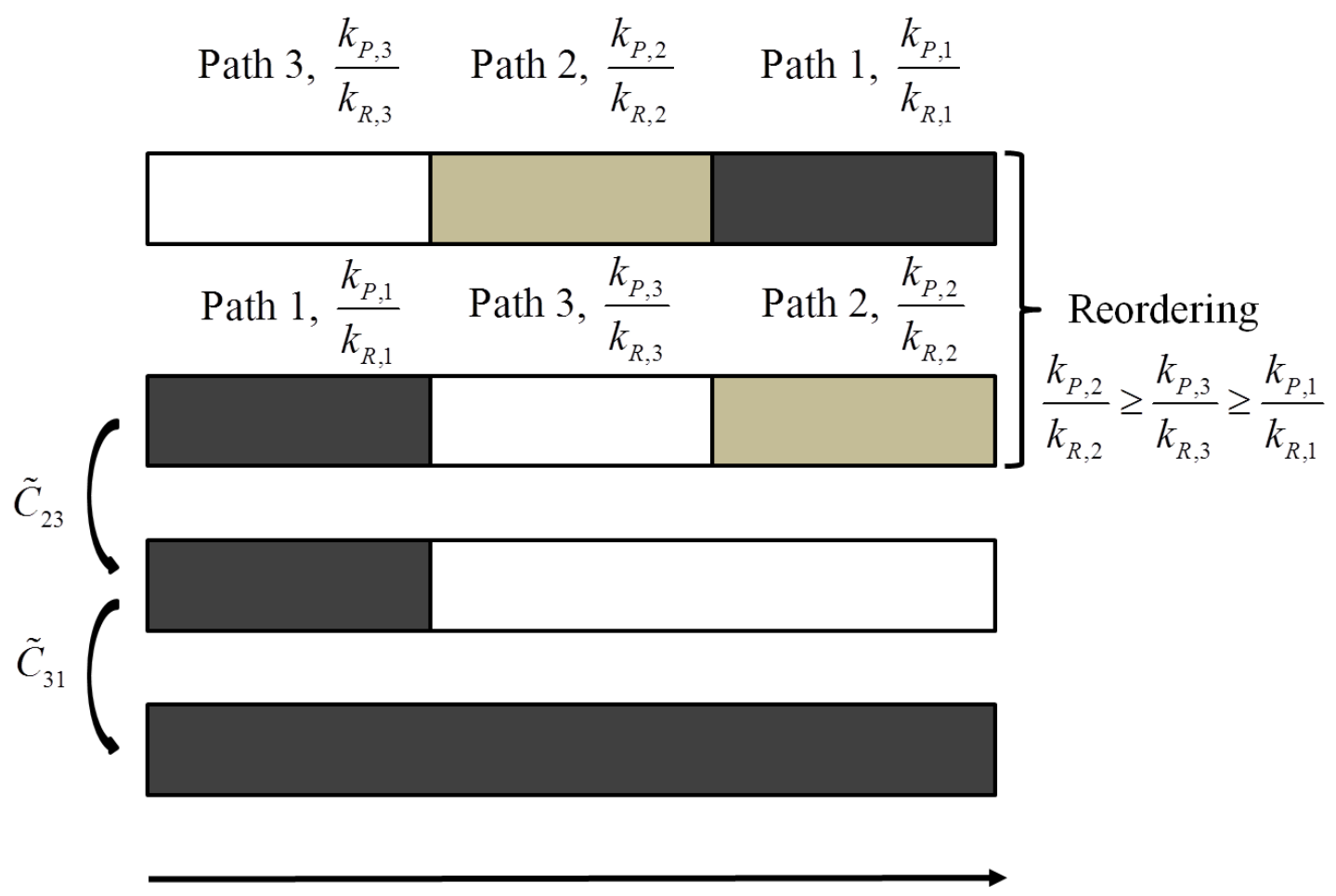

Figure 4-b. LS-ICE method, which consists in applying the ICE schemes to a path reordered with respect to decreasing values of the parameter $\frac{k_{P}}{k_{R}}$ (see text for details). Radiation propagates from left to right. 


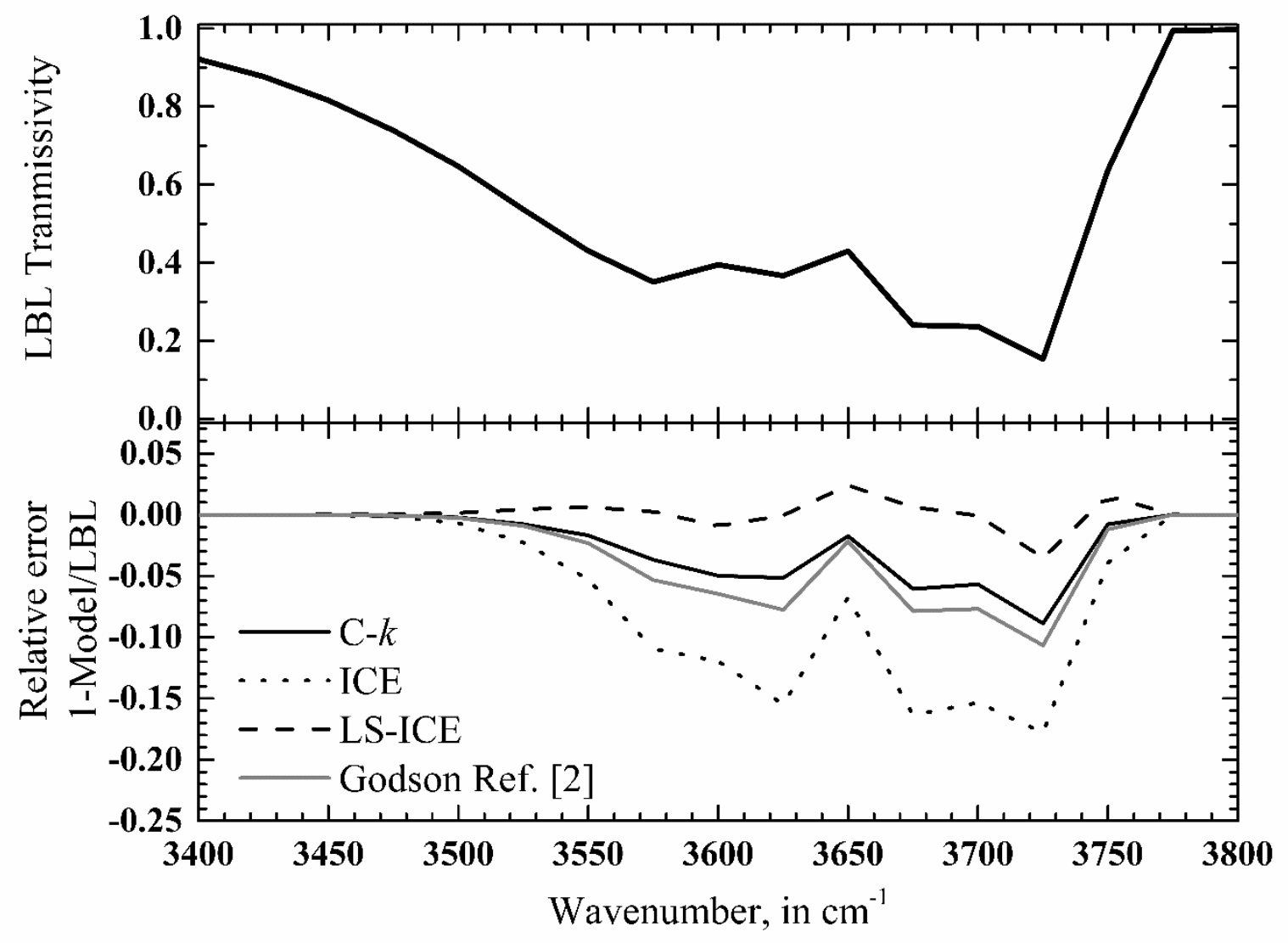

Figure 5. Case $\mathrm{C} 1$ - Narrow band transmissivity for a non-isothermal $\mathrm{CO}_{2}-\mathrm{N}_{2}$ mixture (see text for details) calculated by the LBL, C- $k$ and $\ell$-distribution models with three non-uniform approximations (ICE, LS-ICE and Godson's method). 


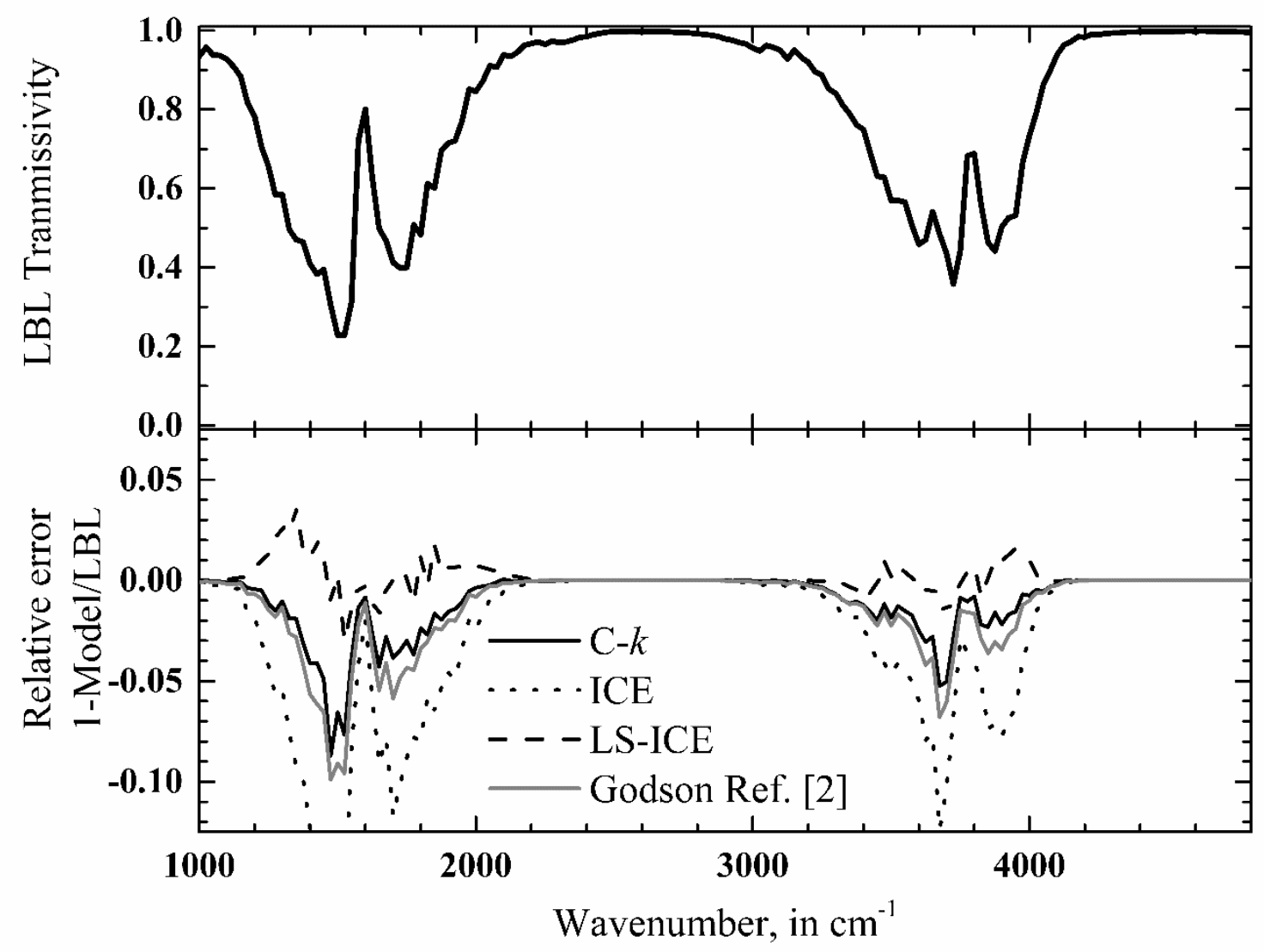

Figure 6. Case $\mathrm{C} 2$ - Narrow band transmissivity for a non-uniform $\mathrm{H}_{2} \mathrm{O}-\mathrm{N}_{2}$ mixture (see text for details) calculated by the LBL, C- $k$ and $\ell$-distribution models with three non-uniform approximations (ICE, LS-ICE and Godson's method). 


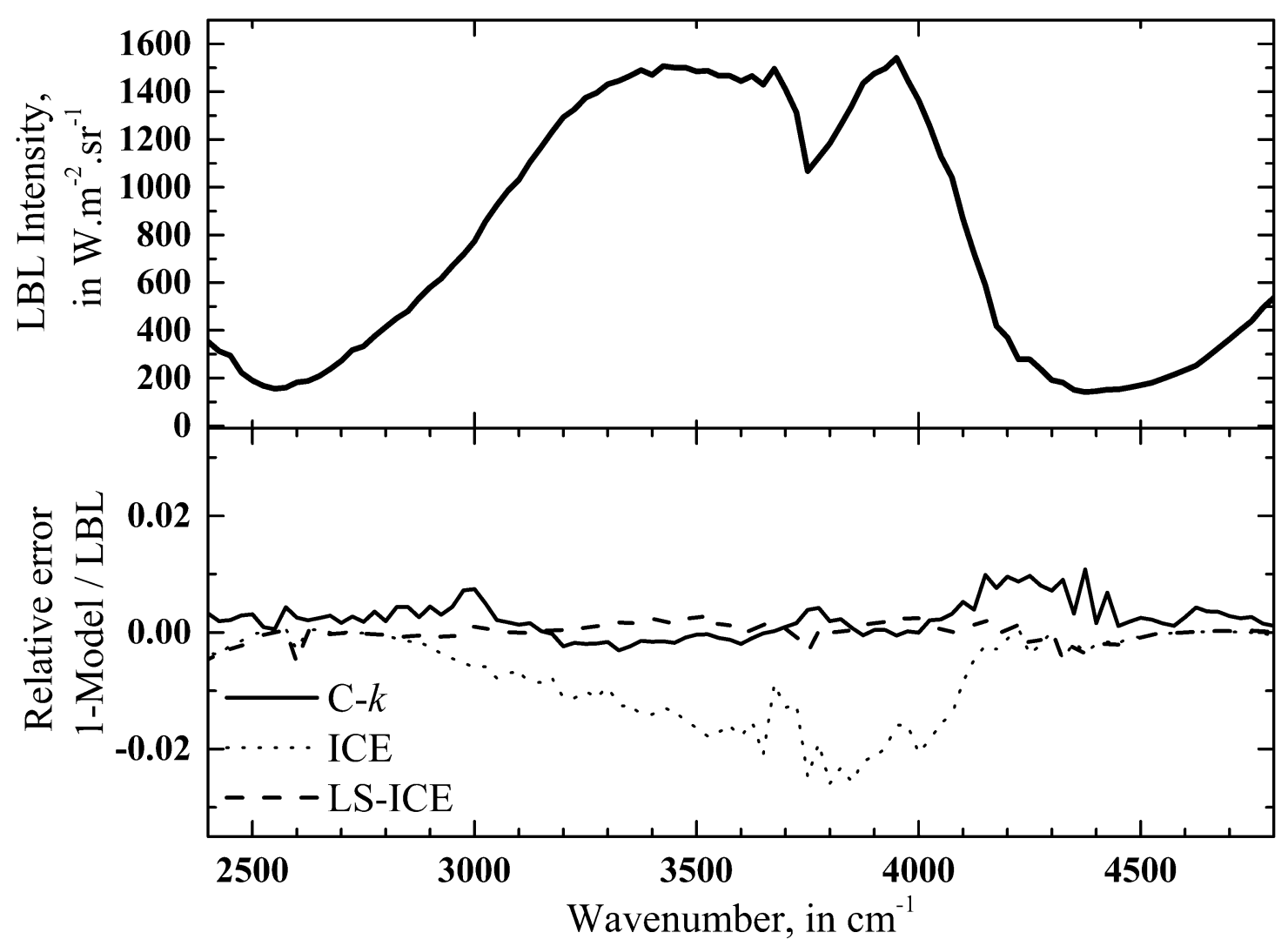

Figure 7. Case C3. Narrow band intensity for a non-uniform $\mathrm{H}_{2} \mathrm{O}-\mathrm{N}_{2}$ mixture (see Table 1 for details) calculated by the LBL, C- $k$ and $\ell$-distribution models with two non-uniform approximations (ICE and LS-ICE). 


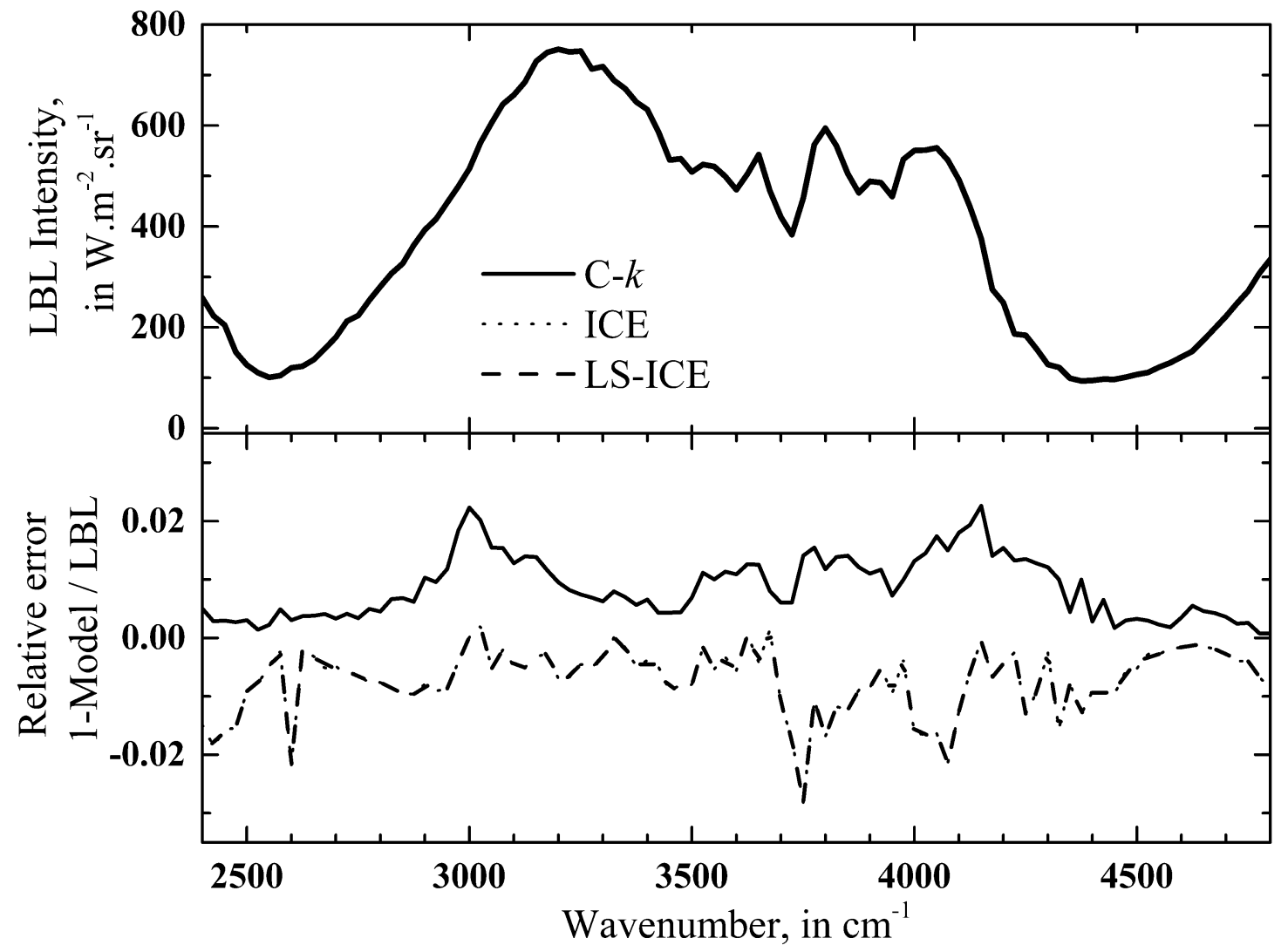

Figure 8. Case $\mathrm{C} 4$. Narrow band intensity for a non-uniform $\mathrm{H}_{2} \mathrm{O}-\mathrm{N}_{2}$ mixture (see Table 1 for details) calculated by the LBL, C- $k$ and $\ell$-distribution models with two non-uniform approximations (ICE and LS-ICE). 


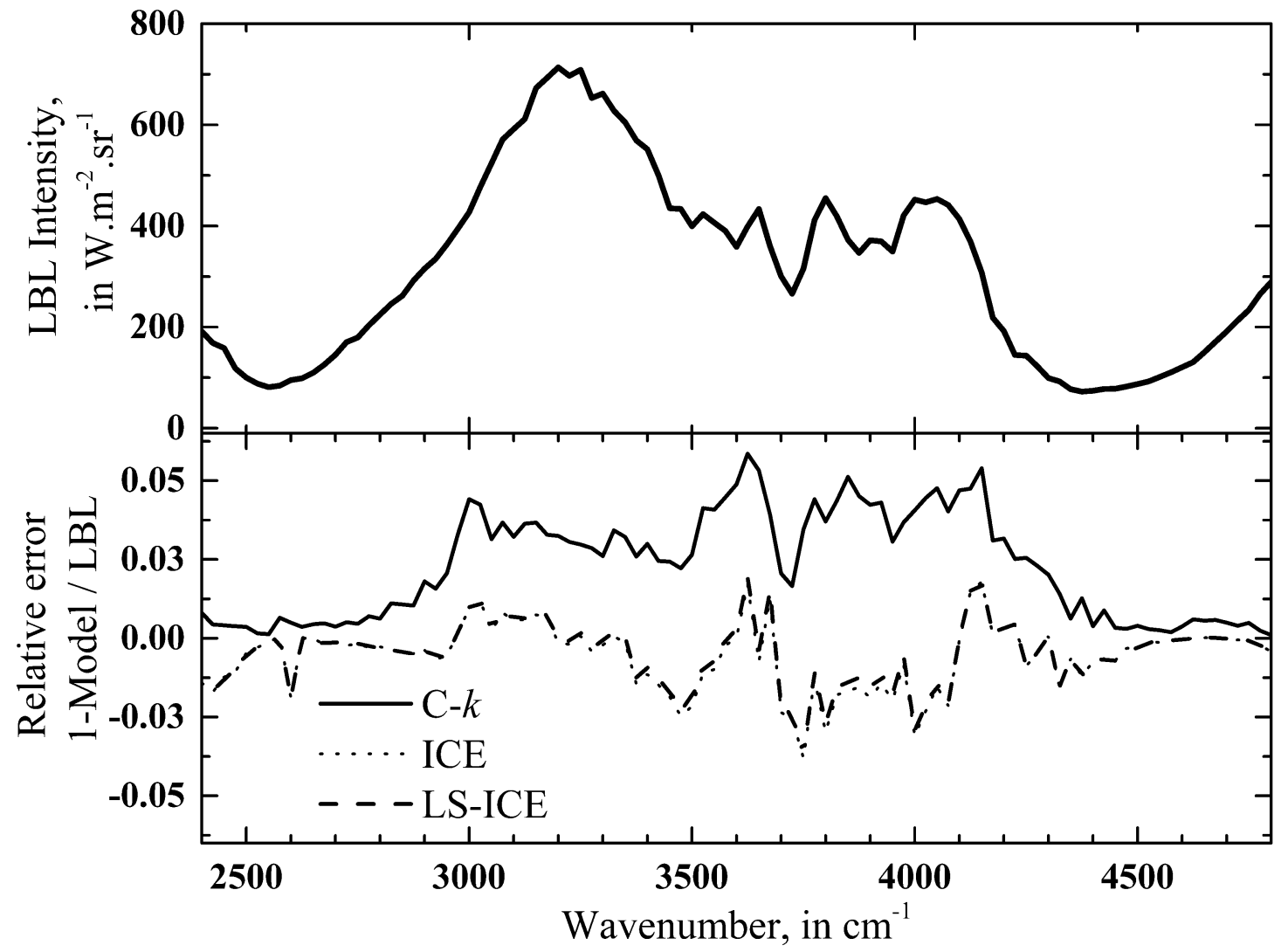

Figure 9. Case $\mathrm{C} 5$. Narrow band intensity for a non-uniform $\mathrm{H}_{2} \mathrm{O}-\mathrm{N}_{2}$ mixture (see Table 1 for details) calculated by the LBL, C- $k$ and $\ell$-distribution models with two non-uniform approximations (ICE and LS-ICE). 


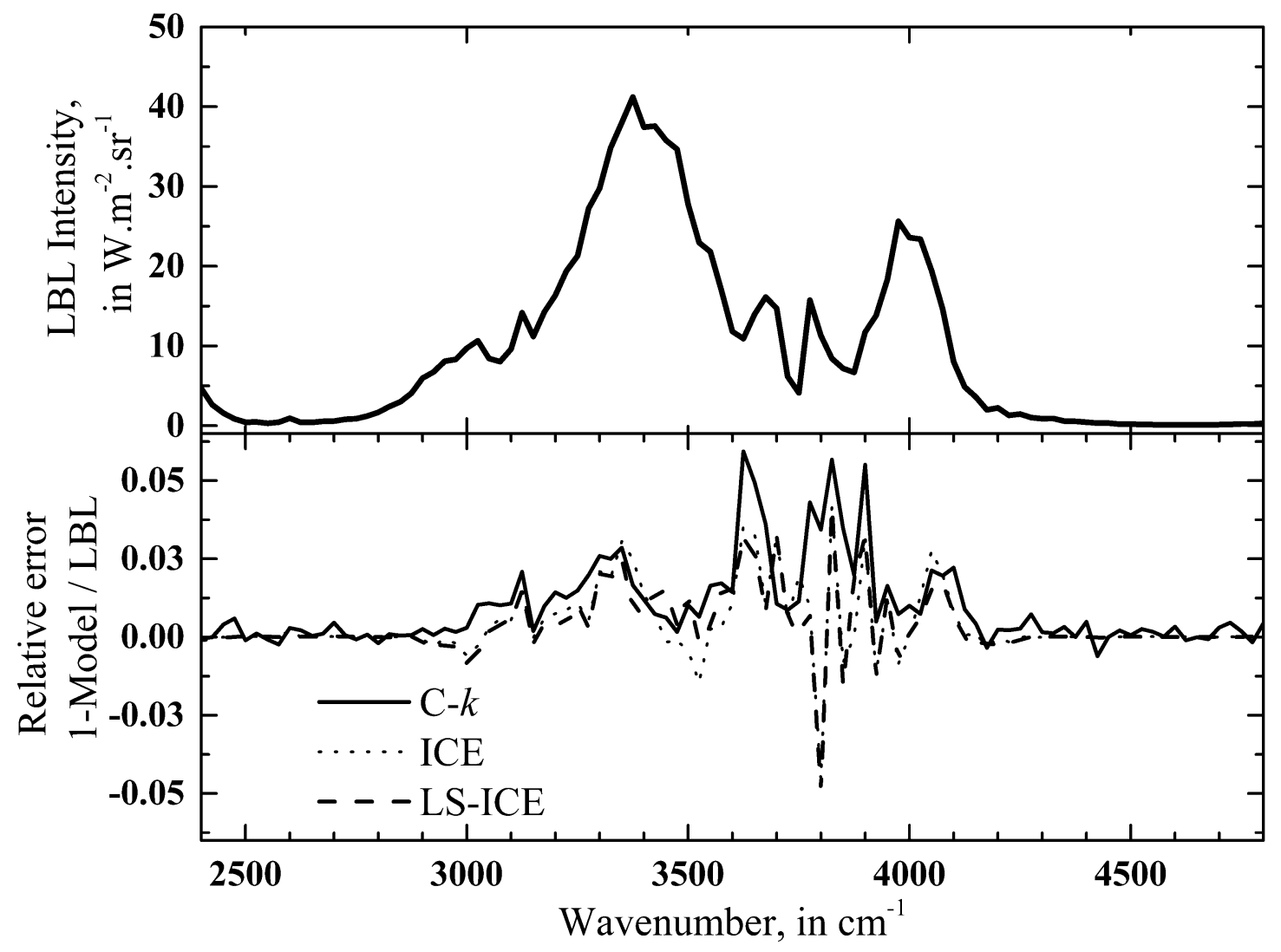

Figure 10. Case C6. Narrow band intensity for a non-uniform $\mathrm{H}_{2} \mathrm{O}-\mathrm{N}_{2}$ mixture (see text for details) calculated by the LBL, C- $k$ and $\ell$-distribution models with two non-uniform approximations (ICE and LS-ICE). 


\section{TABLES}

\begin{tabular}{|c|c|c|c|c|}
\hline Case & Path \#1 & Path \#2 & Path \#3 & Path \#4 \\
\hline C3 & $\begin{array}{c}2480 \mathrm{~K} \\
0.37\end{array}$ & $\begin{array}{c}1220 \mathrm{~K} \\
0.14\end{array}$ & $\begin{array}{c}1180 \mathrm{~K} \\
0.13\end{array}$ & $\begin{array}{c}2480 \mathrm{~K} \\
0.37\end{array}$ \\
& & & & \\
\hline & $1210 \mathrm{~K}$ & $1520 \mathrm{~K}$ & $2000 \mathrm{~K}$ & $2480 \mathrm{~K}$ \\
& 0.14 & 0.18 & 0.26 & 0.37 \\
\hline & $1210 \mathrm{~K}$ & $1210 \mathrm{~K}$ & $1180 \mathrm{~K}$ & $2480 \mathrm{~K}$ \\
& 0.14 & 0.14 & 0.13 & 0.37 \\
\hline
\end{tabular}

Table 1. Parameters used for cases $\mathrm{C} 3-5$ - temperature in $\mathrm{K}$ and $\mathrm{H}_{2} \mathrm{O}$ molar fraction - the length of the gas paths is constant $(150 \mathrm{~cm})$ and the total pressure is $1 \mathrm{~atm}$. 


\begin{tabular}{|c|c|c|c|c|}
\hline Case & LBL & $\begin{array}{c}\text { C- } \boldsymbol{k} \\
(\% / \mathrm{LBL})\end{array}$ & $\begin{array}{c}\text { ICE } \\
(\% / \mathrm{LBL})\end{array}$ & $\begin{array}{c}\text { LS-ICE } \\
(\% / \mathrm{LBL})\end{array}$ \\
\hline \multirow{2}{*}{ C3 } & 155096 & $\begin{array}{c}154787 \\
(0.20)\end{array}$ & $\begin{array}{c}157163 \\
(1.33)\end{array}$ & $\begin{array}{c}155373 \\
(-0.18)\end{array}$ \\
\hline \multirow{2}{*}{ C4 } & 81817 & $\begin{array}{c}81093 \\
(0.88)\end{array}$ & $\begin{array}{c}82969 \\
(-1.41)\end{array}$ & $\begin{array}{c}82969 \\
(-1.41)\end{array}$ \\
\hline \multirow{2}{*}{ C5 } & 67528 & 65916 & 68632 & 68564 \\
& & $(2.38)$ & $(-1.63)$ & $(-1.53)$ \\
\hline \multirow{2}{*}{ C6 } & 3311 & 3268 & 3299 & 3307 \\
& & $(1.30)$ & $(0.36)$ & $(0.12)$ \\
\hline
\end{tabular}

Table 2. Band averaged intensity and relative errors (in \% / LBL reference data) for wide band calculations (see text for details) for Cases C3-6. 


\section{APPENDIX A. Principle of the non-uniform approximations used in the ICE scheme}

The aim of this Appendix is to provide a mathematical derivation of the non-uniform method used in the ICE scheme. The problem is formulated in terms of small gradients of temperature but the same approach can be used for gradients of pressure, species concentrations or both variables at the same time.

We consider the case depicted in Figure 1 and assume that gas spectra in the two thermophysical states are related by the following relationship:

$$
\kappa_{\eta}^{1}=\kappa_{\eta}^{2}+\frac{\partial \kappa_{\eta}^{2}}{\partial T} \cdot \delta T
$$

We further assume that:

$$
\kappa_{\eta}^{2} \gg \frac{\partial \kappa_{\eta}^{2}}{\partial T} \cdot \delta T
$$

The transmissivity of a path of length $L_{1}$ in the gas in state $\phi_{1}$ is, following assumption (A.2):

$$
\tau_{1}^{\Delta \eta}\left(L_{1}\right)=\frac{1}{\Delta \eta} \int_{\Delta \eta} \exp \left(-\kappa_{\eta}^{1} L_{1}\right) d \eta \approx \frac{1}{\Delta \eta} \int_{\Delta \eta} \exp \left(-\kappa_{\eta}^{2} L_{1}\right) \cdot\left[1-\frac{1}{\kappa_{\eta}^{2}} \frac{\partial \kappa_{\eta}^{2}}{\partial T} \cdot \delta T \cdot\left[1-\exp \left(-\kappa_{\eta}^{2} L_{1}\right)\right]\right] d \eta
$$

Eq. (A.3) can be rewritten as:

$$
\tau_{1}^{\Delta \eta}\left(L_{1}\right) \approx \tau_{2}^{\Delta \eta}\left(L_{1}\right)-\frac{1}{\Delta \eta} \int_{\Delta \eta} \exp \left(-\kappa_{\eta}^{2} L_{1}\right) \frac{1}{\kappa_{\eta}^{2}} \frac{\partial \kappa_{\eta}^{2}}{\partial T} \cdot \delta T \cdot\left[1-\exp \left(-\kappa_{\eta}^{2} L_{1}\right)\right] d \eta
$$

The transmissivity of a path $L_{1}+\delta L_{1}, L_{1} \gg \delta L_{1}$ in the gas in the state $\phi_{2}$ can be expanded as: 


$$
\tau_{2}^{\Delta \eta}\left(L_{1}+\delta L_{1}\right) \approx \tau_{2}^{\Delta \eta}\left(L_{1}\right)+\frac{\partial \tau_{2}^{\Delta \eta}\left(L=L_{1}\right)}{\partial L} \cdot \delta L_{1}
$$

Comparing Eq. (A.4) and (A.5) allows proposing the following approximation:

$$
\left\{\begin{array}{l}
\tau_{1}^{\Delta \eta}\left(L_{1}\right) \approx \tau_{2}^{\Delta \eta}\left(L_{1}+\delta L_{T}\right) \\
\delta L_{T}=-\frac{1}{\Delta \eta} \int_{\Delta \eta} \exp \left(-\kappa_{\eta}^{2} L_{1}\right) \frac{1}{\kappa_{\eta}^{2}} \frac{\partial \kappa_{\eta}^{2}}{\partial T} \cdot \delta T \cdot\left[1-\exp \left(-\kappa_{\eta}^{2} L_{1}\right)\right] d \eta \cdot\left[\frac{\partial \tau_{2}^{\Delta \eta}\left(L=L_{1}\right)}{\partial L}\right]^{-1}
\end{array}\right.
$$

viz. small spatial non-uniformities of temperature can be represented as a first approximation by using an equivalent length or by defining an effective scaling coefficient between the gas spectra as $u\left(L_{1}\right)=1+\delta L_{T} / L_{1}$.

The same idea can be used over non-isothermal layers. Indeed, the transmissivity of the nonuniform path $L=L_{1}+L_{2}$ is, making use of Eq. (A.2):

$$
\begin{aligned}
\tau_{12}^{\Delta \eta}\left(L_{1}, L_{2}\right) & =\frac{1}{\Delta \eta} \int_{\Delta \eta} \exp \left(-\kappa_{\eta}^{1} L_{1}-\kappa_{\eta}^{2} L_{2}\right) d \eta \\
& \approx \frac{1}{\Delta \eta} \int_{\Delta \eta} \exp \left(-\kappa_{\eta}^{2} L_{1}-\kappa_{\eta}^{2} L_{2}\right) \cdot\left[1-\frac{1}{\kappa_{\eta}^{2}} \frac{\partial \kappa_{\eta}^{2}}{\partial T} \cdot \delta T \cdot\left[1-\exp \left(-\kappa_{\eta}^{2} L_{1}\right)\right]\right] d \eta
\end{aligned}
$$

Following the same method as in the uniform case, one obtains the following approximation:

$$
\left\{\begin{array}{l}
\tau_{12}^{\Delta \eta}\left(L_{1}, L_{2}\right) \approx \tau_{2}^{\Delta \eta}\left(L_{1}+\delta L_{T}^{\prime}+L_{2}\right) \\
\delta L_{T}^{\prime}=-\frac{1}{\Delta \eta} \int_{\Delta \eta} \exp \left[-\kappa_{\eta}^{2}\left(L_{1}+L_{2}\right)\right] \frac{1}{\kappa_{\eta}^{2}} \frac{\partial \kappa_{\eta}^{2}}{\partial T} \cdot \delta T \cdot\left[1-\exp \left(-\kappa_{\eta}^{2} L_{1}\right)\right] d \eta \cdot\left[\frac{\partial \tau_{2}^{\Delta \eta}\left(L=L_{1}+L_{2}\right)}{\partial L}\right]^{-1}
\end{array}\right.
$$

Then, if we assume: 


$$
\delta L_{T}^{\prime} \approx \delta L_{T}
$$

which appears to be a reasonable assumption for small values of $L_{2}$ (see Eqs. (A.6,A.8)), we obtain eventually the following approximation:

$$
\left\{\begin{array}{l}
\tau_{12}^{\Delta \eta}\left(L_{1}, L_{2}\right) \approx \tau_{2}^{\Delta \eta}\left(L_{1}+L_{2}+\delta L_{T}\right) \\
\delta L_{T}=-\frac{1}{\Delta \eta} \int_{\Delta \eta} \exp \left(-\kappa_{\eta}^{2} L_{1}\right) \frac{1}{\kappa_{\eta}^{2}} \frac{\partial \kappa_{\eta}^{2}}{\partial T} \cdot \delta T \cdot\left[1-\exp \left(-\kappa_{\eta}^{2} L_{1}\right)\right] d \eta \cdot\left[\frac{\partial \tau_{2}^{\Delta \eta}\left(L=L_{1}\right)}{\partial L}\right]^{-1}
\end{array}\right.
$$

The previous equation can be readily reformulated into:

$$
\tau_{12}^{\Delta \eta}\left(L_{1}, L_{2}\right) \approx \tau_{2}^{\Delta \eta}\left[\ell_{2} \circ \tau_{1}^{\Delta \eta}\left(L_{1}\right)+\ell_{2} \circ \tau_{2}^{\Delta \eta}\left(L_{2}\right)\right]=\tau_{2}^{\Delta \eta}\left[\ell_{2} \circ \tau_{1}^{\Delta \eta}\left(L_{1}\right)+L_{2}\right]
$$

where function $\ell_{2}(X), X \in[0,1]$ is defined as the inverse of the transmissivity $\tau_{2}^{\Delta \eta}\left(L_{2}\right)$ over the uniform path $L_{2}$, viz. $\ell_{2}\left[\tau_{2}^{\Delta \eta}\left(L_{2}\right)\right]=L_{2}$. This follows directly from Eq. (A.6) - which corresponds to the implicit equation encountered in the ICE method - rewritten as:

$$
\ell_{2} \circ \tau_{1}^{\Delta \eta}\left(L_{1}\right)=L_{1}+\delta L_{T}
$$


APPENDIX B. Physical analysis of the criterion $\frac{k_{P, 1}}{k_{R, 1}} \geq \frac{k_{P, 2}}{k_{R, 2}}$

In order to better understand why applying the criterion $\frac{k_{P, 1}}{k_{R, 1}} \geq \frac{k_{P, 2}}{k_{R, 2}}$ is likely to improve the model accuracy, we are going to consider here a simple problem.

The ratio $\frac{k_{P}}{k_{R}}$ takes values higher than 1 except when the gas is gray in which case $\frac{k_{P}}{k_{R}}=1$. Let us thus consider the following situation: $\frac{k_{P, 1}}{k_{R, 1}}>\frac{k_{P, 2}}{k_{R, 2}}=1$ for which the gas is gray in column 2 and non-gray in column 1. Eq. (2) becomes:

$$
\begin{aligned}
\tilde{C}_{12}\left(X_{1}, X_{2}\right) & =\frac{1}{\Delta \eta} \int_{\Delta \eta} \exp \left(-\kappa_{\eta}^{2}\left[\ell_{2}\left(X_{1}\right)+\ell_{2}\left(X_{2}\right)\right]\right) d \eta \\
& =\exp \left(-k_{P, 2}\left[\ell_{2}\left(X_{1}\right)+\ell_{2}\left(X_{2}\right)\right]\right)
\end{aligned}
$$

where:

$$
\ell_{2}(X)=-\frac{1}{k_{P, 2}} \ln X
$$

In this case, the true transmissivity:

$$
\begin{aligned}
\tau_{12}^{\Delta \eta}\left(L_{1}, L_{2}\right) & =\frac{1}{\Delta \eta} \int_{\Delta \eta} \exp \left(-\kappa_{\eta}^{1} L_{1}-k_{P, 2} L_{2}\right) d \eta \\
& =\frac{1}{\Delta \eta} \int_{\Delta \eta} \exp \left(-\kappa_{\eta}^{1} L_{1}\right) d \eta \exp \left(-k_{P, 2} L_{2}\right) \\
& =\tau_{1}^{\Delta \eta}\left(L_{1}\right) \tau_{2}^{\Delta \eta}\left(L_{2}\right)
\end{aligned}
$$

coincides exactly with its approximation $\tilde{\tau}_{12}^{\Delta \eta}\left(L_{1}, L_{2}\right)=\tilde{C}_{12}\left[\tau_{1}^{\Delta \eta}\left(L_{1}\right), \tau_{2}^{\Delta \eta}\left(L_{2}\right)\right]$ since: 


$$
\begin{aligned}
\tilde{C}_{12}\left[\tau_{1}^{\Delta \eta}\left(L_{1}\right), \tau_{2}^{\Delta \eta}\left(L_{2}\right)\right] & =\exp \left(-k_{P, 2}\left[\ell_{2}\left(\tau_{1}^{\Delta \eta}\left(L_{1}\right)\right)+\ell_{2}\left(\tau_{2}^{\Delta \eta}\left(L_{2}\right)\right)\right]\right) \\
& =\exp \left(-k_{P, 2}\left[-\frac{\ln \left(\tau_{1}^{\Delta \eta}\left(L_{1}\right)\right)}{k_{P, 2}}-\frac{\ln \left(\tau_{2}^{\Delta \eta}\left(L_{2}\right)\right)}{k_{P, 2}}\right]\right) \\
& =\tau_{1}^{\Delta \eta}\left(L_{1}\right) \tau_{2}^{\Delta \eta}\left(L_{2}\right)
\end{aligned}
$$

This shows that if the gas in the second column is gray, the solution provided by Eq. (2), which corresponds to a treatment of the non-uniform path in the direction defined by the criterion $\frac{k_{P, 1}}{k_{R, 1}} \geq \frac{k_{P, 2}}{k_{R, 2}}$, is exact. In the same case, it is impossible to provide any detail about the accuracy of Eq. (5), i.e. if the calculation is done in the other direction. 


\section{APPENDIX C. Elements of Archimedean copula theory}

The objective of this section is not to provide a comprehensive depiction of copula's theory. More details about copulas can be found in Refs. [15-17].

\section{C.1. Introduction to Archimedean copulas}

The concept of copula was introduced by Sklar in Ref. [18]. It has received many interest due to the fact that any multivariate distribution function can be obtained by combining the distribution function of each of the variables taken separately (the marginals) with a copula: this result is known as Sklar's theorem. Copulas are encountered in a large range of problems in statistical finance, theoretical physics, bio-statistics, etc.

A $n$-copula $C$ is [15-17] a multivariate distribution function defined over the hypercube, $[0,1]^{n}$, with uniform marginals. It defines a map from $[0,1]^{n} \rightarrow[0,1]$. In the bi-dimensional case, a 2copula with continuous derivatives has the properties set by Eqs. (B.6) below. More general definitions together with extensions to dimensions higher than 2 can be found in Ref. [15].

There is a wide range of distinct copulas. A compendium of the existing ones is given, for instance, in Ref. [15]. Among them, the class of Archimedean copulas is among the most popular.

The key element in the definition of an Archimedean copula is its generator, $\varphi . \varphi$ is a continuous strictly decreasing function from $[0,+\infty) \rightarrow[0,1]$ such that: $1 / \varphi(0)=1,2 / \varphi(+\infty)=0,3 / \varphi$ is completely monotonic, which means that it has derivative of all order and that these derivatives alternate in sign, i.e.:

$$
(-1)^{n} \frac{d^{n} \varphi(X)}{d X^{n}} \geq 0, n=0,1,2, . ., X \in[0,+\infty]
$$


Once such a generator is chosen, the corresponding copula can be written as:

$$
C_{\varphi}(X, Y)=\varphi\left[\varphi^{-1}(X)+\varphi^{-1}(Y)\right]
$$

One property of Archimedean copulas is that they are symmetric, viz.: $C_{\varphi}(X, Y)=C_{\varphi}(Y, X)$. This property, called exchangeability [19], strongly limits the type of statistical dependences that one can model using this class of functions. Consequently, various extensions of Archimedean copulas were proposed to take into account the non-exchangeability of the variables. One of them consists in nesting 2-copulas in order to produce asymmetric dependency structures. The nesting process mostly consists in defining multivariate copulas by composing copulas with other copulas. A nested Archimedean copula takes the following form:

$$
\begin{aligned}
C_{1 \ldots n}\left(X_{1}, X_{2}, . ., X_{n}\right) & =\varphi_{n}\left[\varphi_{n}^{-1}\left(X_{n}\right)+\varphi_{n}^{-1} \circ C_{1 \ldots n-1}\left(X_{1}, X_{2}, . ., X_{n-1}\right)\right] \\
& =\varphi_{n}\left[\varphi_{n}^{-1}\left(X_{n}\right)+\varphi_{n}^{-1} \circ \varphi_{n-1}\left[\varphi_{n-1}^{-1}\left(X_{n-1}\right)+\varphi_{n-1}^{-1} \circ C_{1 \ldots n-2}\left(X_{1}, X_{2}, . ., X_{n-2}\right)\right]\right] \\
& =\ldots
\end{aligned}
$$

in which $\varphi_{n}, n=1, . ., N$ is a sequence of generators.

More details about nested Archimedean copulas can be found in Ref. [19].

However, although the nesting process is simple to understand (and to apply if the generators are defined analytically, for instance), it does not ensure the multivariate function generated by composing copulas with copulas to be a copula itself.

Nevertheless, it can be shown (see for instance Ref. [17]) that if the coupling functions defined as $\omega_{n-1, n}=\varphi_{n}^{-1} \circ \varphi_{n-1}$ have a completely monotonic derivative, then the nested structure is ensured to be a copula. If this condition (called the compatibility condition, [19]) is fulfilled, the generator 
$\varphi_{n-1}$ and $\varphi_{n}$ are said to be compatible. In this case, the nested copula is called Levy subordinated (this terminology was introduced in Ref. [20]. It follows from the fact that the compatibility condition is satisfied if and only if the coupling function takes the form of a Levy subordinator, viz. its derivative can be written as a Laplace transform).

\section{C.2. Application within the frame of gas radiation modeling}

The aim of $\tilde{C}_{12}$ and $\tilde{C}_{21}$, as defined by Eq. (3) and Eq. (5), is to provide an approximation of the following function, written $C_{12}$, whose definition arises directly from Eq. (1):

$$
\tau_{12}^{\Delta \eta}\left(L_{1}, L_{2}\right)=C_{12}\left[\tau_{1}\left(L_{1}\right), \tau_{2}\left(L_{2}\right)\right]
$$

where:

$$
C_{12}\left(X_{1}, X_{2}\right)=\frac{1}{\Delta \eta} \int_{\Delta \eta} \exp \left[-\kappa_{\eta}^{1} \ell_{1}\left(X_{1}\right)-\kappa_{\eta}^{2} \ell_{2}\left(X_{2}\right)\right] d \eta
$$

Eqs. (C.4, C.5) provide the exact non-uniform transmissivity as they just consist of a rewriting of Eq. (1).

Function $C_{12}$ has the following properties (the first two equations are written here for the first variable $X_{1}$ only. Symmetrical relationships can be found for the second variable $X_{2}$ ):

$$
\begin{gathered}
C_{12}\left(X_{1}=1, X_{2}\right)=\frac{1}{\Delta \eta} \int_{\Delta \eta} \exp \left[-\kappa_{\eta}^{2} \ell_{2}\left(X_{2}\right)\right] d \eta=X_{2} \\
C_{12}\left(X_{1}=0, X_{2}\right)=0 \\
\frac{\partial^{2} C_{12}\left(X_{1}, X_{2}\right)}{\partial X_{1} \partial X_{2}}=\frac{\partial \ell_{1}\left(X_{1}\right)}{\partial X_{1}} \frac{\partial \ell_{2}\left(X_{2}\right)}{\partial X_{2}} \frac{1}{\Delta \eta} \int_{\Delta \eta} \kappa_{\eta}^{1} \kappa_{\eta}^{2} \exp \left[-\kappa_{\eta}^{1} \ell_{1}\left(X_{1}\right)-\kappa_{\eta}^{2} \ell_{2}\left(X_{2}\right)\right] d \eta \geq 0
\end{gathered}
$$


Eqs. (C.6) follow directly from the definition of functions $\ell_{i}, i=1,2$ as the inverse of the transmissivities $\tau_{i}^{\Delta \eta}, i=1,2$. Indeed, according to this definition, we have:

$$
\begin{gathered}
\ell_{i}\left(X_{i}=1\right)=0 \\
\ell_{i}\left(X_{i}=0\right)=+\infty \\
\frac{\partial \ell_{i}\left(X_{i}\right)}{\partial X_{i}}=-\left[\frac{1}{\Delta \eta} \int_{\Delta \eta} \kappa_{\eta}^{i} \exp \left[-\kappa_{\eta}^{i} \ell_{i}\left(X_{i}\right)\right] d \eta\right]^{-1} \leq 0
\end{gathered}
$$

Eq. (C.7-a) is used to justify Eq. (C.6-a), Eq. (C.7-b) is involved in the derivation of Eq. (C.6-b). The inequality Eq. (C.6-c) follows from Eq. (C.7-c).

As transmissivities are distribution functions, the set of Eqs. (C.6) shows that function $C_{12}$ is a copula. As a consequence of Sklar's theorem, $\tau_{12}^{\Delta \eta}\left(L_{1}, L_{2}\right)$ is a joint distribution function: this is the main principle of the $\ell$-distribution approach.

\section{C.3. Conditions for the function $\tilde{C}$ to be Levy subordinated}

Following the previous mathematical section, we first notice that transmissivities are completely monotonic functions. This follows directly from:

$$
(-1)^{n} \frac{d^{n} \tau^{\Delta \eta}(L)}{d L^{n}}=\frac{1}{\Delta \eta} \int_{\Delta \eta}\left(\kappa_{\eta}\right)^{n} \exp \left(-\kappa_{\eta} L\right) d \eta \geq 0, n=0,1,2, . ., L \in[0,+\infty)
$$

The completely monotonic property of transmissivities has many useful applications. One of them is a pure theoretical justification of the $k$-distribution approach through the use of the HaussdorfBernstein-Widder theorem. Further comments can be found for instance in Ref. [21].

Accordingly, transmissivities can be used as Archimedean generators. The corresponding copulas then take the following form, considering for instance $\varphi=\tau_{1}^{\Delta \eta}$ :

$$
C_{\tau_{1}^{\Delta \eta}}(X, Y)=\tau_{1}^{\Delta \eta}\left[\ell_{1}(X)+\ell_{1}(Y)\right]=\tilde{C}_{12}(X, Y)
$$


or equivalently $\varphi=\tau_{2}^{\Delta \eta}$

$$
C_{\tau_{2}^{\Delta \eta}}(X, Y)=\tau_{2}^{\Delta \eta}\left[\ell_{2}(X)+\ell_{2}(Y)\right]=\tilde{C}_{21}(X, Y)
$$

The ICE scheme processes by replacing step by step couples of contiguous uniform layers along the non-uniform path by equivalent uniform ones. This process can be mathematically formulated in terms of nested Archimedean copulas, viz. it can be written in the same form as Eq. (C.3). Accordingly, this means that in order to ensure the transmissivity at the output of the non-uniform path to be a distribution, the uniform paths need to be ordered in such a way that the compatibility condition is fulfilled at each step along the propagation scheme. This is the case, following the results provided in the paper, as soon as the non-uniform path is reordered with respect to decreasing values of the ratio $\frac{k_{P}}{k_{R}}$ since it ensures the compatibility condition Eq. (12) to be correct.

Finally, one can notice that in the $n$-dimensional case, ensuring the approximate model $\tilde{C}_{1 . . n}$ of $C_{1 . . n}$ (as given in a form equivalent to (C.3)) to be copula guarantees one fundamental property of transmissivities of non-uniform path which is: $\partial^{n} \tau_{1 . . n}^{\Delta \eta}\left(L_{1}, . ., L_{n}\right) / \partial L_{1} . . \partial L_{n}$ and $\prod_{i=1}^{n} \partial \tau_{i}^{\Delta \eta}\left(L_{i}\right) / \partial L_{i}$ have the same signs i.e. both of them are positive if $n$ is odd, they are negative if $n$ is even. 